\title{
NUMERICAL MODELING OF HF SKYWAVE RADIATION FROM ANTENNAS IN IRREGULAR TERRAIN
}

\author{
G. J. Burke
}

November, 1990

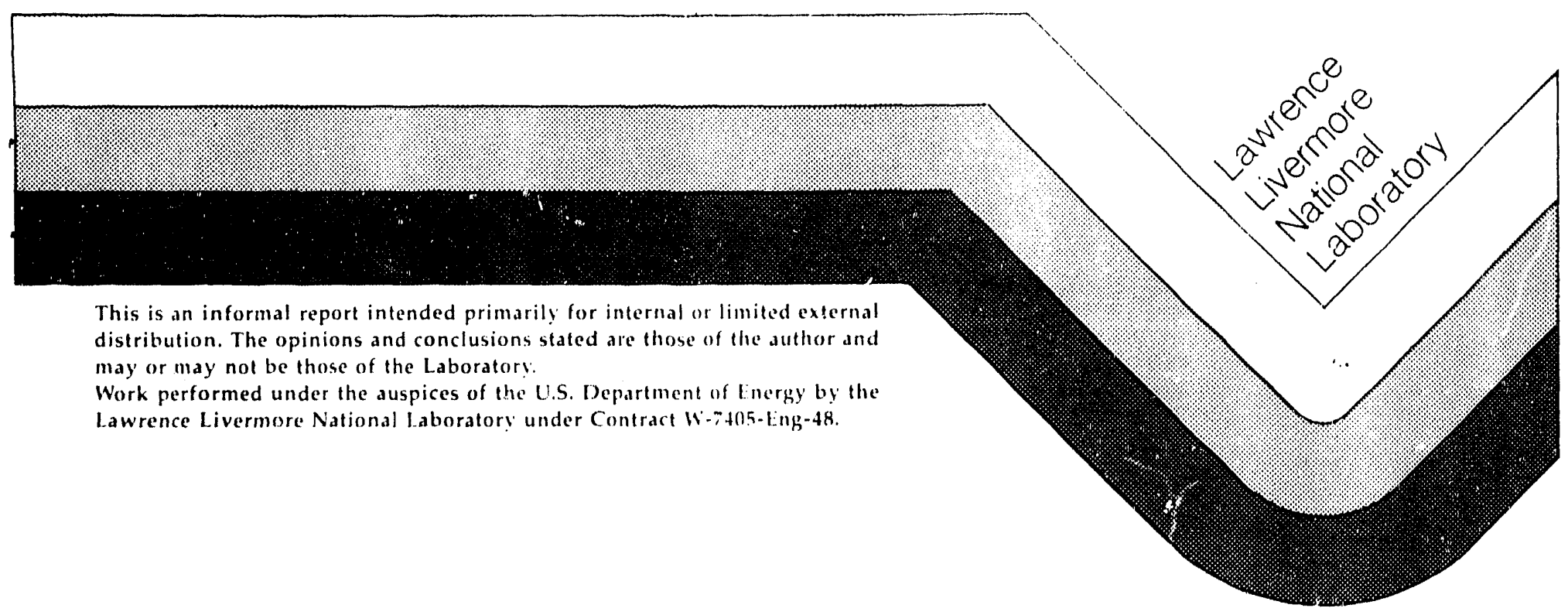

\section{MASTER}




\title{
DISCLAIMER
}

This document was prepared as an account of work sponsored by an agency of the United States Government. Neither the United Siales Government nor the University of California nor any of their employees, makes any warranty, express or implied, or assumes any legal liability or responsibility for the accuracy, completeness, or usefulness of any information, apparatus, producl, or process disclosed, or represents that its use would not infringe privately owned rights. Reference herein to any specific con. niercial products, process, or service by Irade name, trademark, manufacturer, or otherwise, does not necessarily constitute or imf its endorsement, recommendation, or favoring by the United States Government or the University of Calif mus. The views and opinions of authors expressed herein do not necessarily state or reflect those of the United States Government or the University of California, and shall not be used for advertising or product endorsement purposes.

\author{
This report has been reproduced \\ directly from the best abilable copy: \\ Available to DOE and DOE conitactors from the \\ Office of Scientific and Technical Information \\ P.O. Hov 62, Oak Ridge, TN 37831 \\ I'rices available from 1615) 576-8401, FTS 626-8401. \\ Available to the public from the \\ National Technical Information Service \\ U.S. Department of Commerse \\ 5265 l'orl Royal Kd. \\ Springfield, VA 2216
}

Price

Code

A01

Papercopy Prices

A02

A03

A 14

A05

A06

A07

A08

A09

A10

A 11

A12

A13

A14

A 15

A16

A 17

A 18

A 19

A 20

A 21

A 222

A23

A 24

A25

A99
Page

Range

Microfiche

1- 10

11- 50

51- 75

76-100

101-125

126-150

151-175

176-200

201-225

226-250

251-275

276-300

301-325

326-350

351-375

376-400

401-425

426-450

451-475

476-500

$501-52$.

526-550

551-575

576-600

$601 \&$ UP 


\title{
NUMERICAL MODELING OF HF SKYWAVE RADIATION FROM ANTENNAS IN IRREGULAR TERRAIN
}

\begin{abstract}
The problem of computing the radiation pattern of an HF antenna sited in irregular terrain was investigated. The primary interest is in antennas for skywave communication, however ionospheric models were not considered. Several methods for modeling terrain effects are briefly reviewed. A geometrical optics model for arbitrary terrain is developed and results are compared with published results from solution of a Volterra integral equation for scattering by a Gaussian ridge.
\end{abstract}

\section{Introduction}

The effect of irregular terrain on antenna radiation is one remaining part of the $\mathrm{HF}$ communications link for which no general modeling capability is available. A large amount of work has been done on terrain modeling, and prediction methods have been demonstrated for a number of specific cases. The lack of more general models appears to be the result of the difficulty of the problem and the variety of conditions that may occur. An antenna might be mounted near hills or a cliff, on the top or side of a hill, or in a valley. Depending on the angle of radiation the field may involve scattering and diffraction from single or multiple points and surface wave propagation. Terrain features may vary in size from less than a wavelength to many wavelengths over the HF band, and surface roughness may also be an important factor. Hence, analysis of general three dimensional terrain models can exceed the capabilities of even modern computers and modeling codes. In order to develop a general terrain modeling capability it appears necessary to combine several modeling techniques.

This repart covers work on the first phase of a project for the U.S. Navy to develop and apply models for texrain effects in HF communications involving skywave. Candidate techniques are briefly reviewed with some references to previous work. As an initial approach to the problem, a program for modeling terrain was developed using geometrical optics (GO), since this technique is relatively simple and allows three dimensional terrain models. A code for applying GO on arbitrary surfaces was developed using the ray launching method of Mittra and Rushdi [1]. Ray launching is implemented to handle concave as well as convex surfaces and finite conductivity of the ground. The GO model is not suitable for computing the fields at low radiation angles or in shadow regions since surface wave and diffraction are neglected. However, it should give a reasonable estimate of field in illuminated regions, and will provide a point of comparison with other more complete models that may be investigated in the future. 
Our primary interest is in antennas for skywave communication. However, models for the ionosphere are not considered since such models are already available, and ionospheric. reflection can be treated separately from the effects of terrain in the vicinity of the antenna.

The problem of determining the radiation from antennas in irregular terrain is closely linked to that of computing the radiation pattern of the antennas. General purpose computer programs, such as the Numerical Electromagnetics Code (NEC) [2, 3] and MININEC (4) have been developed and widely used for modeling HF antennas. Typically these programs can include the influence of a flat ground with finite conductivity and will determine the antenna impedance and efficiency in this envirorment, as well as computing the radiated field, gain and ground wave strength. Except for simple geometrical-optics models for one or more cliffs in otherwise flat ground, these codes offer no capability for modeling the effects of terrain features on radiation. However, in most cases antennas used in irregular terrain will be sited so that interaction with the terrain does not significantly change the current distribution from when the antenna is on flat grcund. When this is true, the currents determined by codes such as NEC for flat ground can be used as a radiation source in irregular terrain. All that is needed is to reorient the current elements relative to the normal to the terrain at the antenna site and compute the radiation in the presence of the terrain. A transformation for orienting the antenna is given in an appendix to this report.

Several possible techniques for modeling terrain effects are summarized below. The ray launching method, that has been used in developing a computer code for applying GO on arbitrary surfaces, is then described in detail. Results of the GO model are shown for scattering from a Gaussian ridge and compared with results obtained by Berry [5] who solved a Volterra integral equation. The Volterra integral equation solution, particularly as later developed by Ott $[6,7]$, offers a powerful means for modeling terrain with diffraction and surface wave included, and may be investigated in the future as an alternative model for situations where $\mathrm{GO}$ is not adequate.

\section{Terrain Modeling Techniques}

A number of different approaches have been used for modeling terrain effects. Some, such as reflection and knife edge diffraction have been around since the early days of radio. Others, such as integral and differential equation models, involve a heavy computational burden requiring modern computers. Factors affecting the choice of a model include the terrain type, frequency, whether the radiation angle is to the sky or grazing the ground and whether the source is highly directional or illuminates a large area of terrain. Some candidate techniques for modeling terrain effects are summarized below. This is far from a complete listing of references on the subject, but includes some typical work.

\section{Geometrical Optics}

Geometrical optics (GO) provides a conceptually simple model for scattering from terrain. For a flat ground it can be implemented by computing the field of the image 
of the source antenna and a reflection coefficient for the ground, as is now done in the Numerical Electromagnetics Code NEC. With curved surfaces the GO model becomes more complicated, since ray tracing is considerably more difficult, and additional factors must be considered such as the change in ray-tube divergence and possibly muitiple reflections. The GO rnodel can include the effect of finite ground conductivity, and three dimensional terrain models are practical. GO can be expected to give a reasonable estimate of the field in illuminated regions which do not involve ruys that graze the terrain surface. Lack of surface wave makes the model invalid for low radiation angles and may result in under estimating the field scattered by a hill when the source is moderately distant. Without diffraction or surface wave, the model gives zero field in shadow regions.

The principles of $\mathrm{GO}$ are described in $[8,9,10]$. An application of $\mathrm{GO}$ for propagation over earth is discussed in [11], and a model for arbitrary terrain is developed here.

\section{Geometrical Theory of Diffraction}

The Geometrical Theory of Diffraction (GTD) $[12,13,14]$ combines GO with diffraction terms for edges and curved surfaces. With edge diffraction included, a curved surface such as a hill might reasonably be modeled, for scattering calculations, as a collection of flat plates, since the diffracted field will fill the gaps in the reflected field. Diffracted field would also enter some of the shadow regions, although multiple edge diffraction or creeping wave terms are needed to completely fill the shadows. The absence of a surface wave would still limit the accuracy for finitely conducting surfaces at $\mathrm{HF}$.

Knnife edge diffraction models have often been used for propagation over ridges [15]. Luebbers $[16,17]$ has applied modern GTD techniques to this problem, using wedge diffraction formulas modified for finite conductivity and surface roughness and including reflected rays. These models are generally limited to UHF and higher frequencies due to the lack of surface wave, but they might be applicable at HF in cases where ray paths do not graze the surface. Wait $[18,19]$ derived formulas for diffraction of the ground wave by idealized terrain features such as a knife-edge, step and cylindrical hill.

One reason for interest in GTD is the availability of general purpose GTD codes. The Basic Scattering Code (NEC-BSC) [20, 21, 22] can model structures with multiple flat plates, elliptic cylinders and other curved surfaces. The solution includes second order reflection, first order diffraction and reflection-diffraction terms. A creeping wave is included for the elliptic cylinder. NEC-BSC will model a finitely conducting, infinite ground plane, and has a limited capability for modeling thin dielectric plates. The code manual states that a source must be at least a wavelength from a dielectric plate, and the incidence angle from the normal should be less than about 60 degrees since surface waves are not included. The suitability of dielectric plates for modeling terrain is unclear. However, NEC-BSC might be considered for modeling terrain with perfectly conducting plates, particularly at low frequencies where the surface impedance of the ground is low. 
Surface Integration

Several techniques for modeling terrain involve the computation of scattered field from integrals over sources on the terrain surface. Alternatively, the perturbation of the field caused by terrain features, from that with a flat ground, may be obtained by application of the compensation theorem [23]. If the sources are taken to be the surface fields determined by GO, the method resembles physical optics. Physical optics avoids the caustics encountered in GO and may give a reasonable result for field in shadow regions if the obstacle is not too large. The problems of finding specular points and computing divergence factors in GO are traded for a possibly large amount of integrating. V. Liepa [24] demonstrated the use of physical optics for computing the field scattered by a perfectly conducting semicylindrical boss on a surface. This technique can be extended to finitely conducting surfaces by employing the surface impedance approximation.

Page and Monteath [25] computed the effect of small surface irregularities by integrating over the perturbed surface with the surface current assumed to be the same as for a flat site. They compare their results with measurements for an antenna on a plateau a quarter wavelength high and one wavelength in extent.

Volterra Integra! Equation

Rather than using the GO incident fields or assuming a surface current, a more accurate evaluation of the surface fields over irregular terrain is possible by solving an integral equation. Hufford [26] derived a Volterra integral equation for the attenuation of a wave over inhomogeneous irreguiar terrain, starting with Green's theorem and using the free space field as an elementary function. In deriving this equation it is assumed that the terrain varies only with distance from the source and backward scattering is neglected. Ott and Berry $[6,7]$ developed an alternative integral equation, using a modified Sommerfeld attenuation function as an elementary function. This alternative equation is more easily solved than Hufford's and has been used extensively for computing the surface wave along communication paths involving inhomogeneous, irregular terrain $[7,27,28]$. Alternative integral equations have also been derived by $\mathrm{Wu}$, et al. [29] and Bevensee [30]. Bevensee extended the analysis from a point source to a finite current distribution.

The computation of the radiated field by integrating the surface fields obtained from a Volterra integral equation is demonstrated in [5] and [31]. Since the integral equation solution includes effects of reflection and diffraction at surface irregularities, as well as surface wave propagation, the result obtained for skywave should be more accurate than that obtained by physical optics or assumed currents. However, the integral equation is limited to two dimensional terrain models, constant in the direction transverse to the direction of propagation, and neglects backward reflection.

An integral equation similar to Hufford's was derived by Monteath [32] stanting from the Compensation Theorem. This equation was derived for flat ground with varying electrical parameters. It has been used to compute the signal received at varying distances 
from a coast line for ground wave propagation and skywave. Results in [33, 34] show the effect of a coast line extending inland over $50 \mathrm{~km}$ for a $845 \mathrm{kHz}$ skywave signal arriving at 4.3 degrees elevation. The computed results are compared with measurements of Knight and Thoday [35].

\section{Frequency Domain Integral Equations}

The moment-method solution of an integral equation for surface currents can provide a model for irregular terrain. Integral equations can be based on electric, magnetic or combined fields [36] and may involve surface or volume currents. Such models can be very accurate, but computation time limits the frequency and the extent of terrain modeled. For large models, the solution time would increase as the cube of the number of unknowns in the model, or as the sixth power of surface area in square wavelengths. The magnetic field integral equation is relatively easy to solve, but is limited to perfectly conducting closed surfaces. The electric field integral equation requires more elaborate solution methods [ 37 , 38 ] but can model finitely conducting and open, thin surfaces.

Interest in integral equation models is prompted by the current availability of computer codes. Corry and Lane [39] used the magnetic-field integral equation model in NEC to model mountainous terrain up to $5.5 \mathrm{MHz}$. Another option with NEC would be to model the terrain with a finitely conducting wire mesh, using the thin-wire electric field equation.

\section{Finite Difference Time Domain Solution}

Finite-difference time domain (FDTD) codes solve Maxwell's curl equations over a spatial grid, with central differencing for the derivatives of $E$ and $H$, using the Yee algorithm [40]. This technique is capable of accurate modeling of terrain, including inhomogeneous ground. Like the integral equation solution, FDTD is limited by" computation time and storage requirements. Generally these codes are run on large, fasic computers. FDTD has an advantage over integral equations for electrically large models, since it does not require solution of a matrix equation. The size of a FDTD model for terrain would depend on the size of terrain features relative to wavelength and also on where the outer boundary terminating the grid could be placed. Models covering several $\mathrm{km}$ of terrain at $\mathrm{HF}$ appear practical, although it may be necessary to use a two dimensional model to reduce the grid size. Also, the effects of dispersion in the solution grid may become significant over long distances. The time domain solution can provide a wide-band response, although the usefulness of such data may be limited if the ground parameters change substantially over the band.

A FDTD code, TSAR [41], has been developed at Lawrence Livermore National Laboratory. This code includes a CAD program for model generation, display and postprocessing. TSAR appears to have considerable potential in terrain modeling applications, either as a primary model or for validation of other techniques. 


\section{Development of a Geometrical Optics Model for Terrain}

A computer code was developed on this project to apply GO on arbitrary surfaces with finite conductivity. The development of this model is described below and results, showing some uses and limitations of GO for modeling terrain are included in the next section.

In the conventional application of GO to reflection from a surface the surface is searched for specular points contributing to the field at the evaluation point. When a specular point $\vec{Q}_{R}$ is found for the evaluation point $\vec{P}$, the field that the ray produces at the evaluation point is computed as [9]

$$
\vec{E}^{r}(s)=\underbrace{\vec{E}^{i}\left(\vec{Q}_{R}\right)}_{\begin{array}{c}
\text { Field at } \\
\text { reflection }
\end{array}} \cdot \underbrace{\overline{\bar{R}}}_{\begin{array}{c}
\text { Reflection } \\
\text { coefficient }
\end{array}} \underbrace{\sqrt{\frac{\rho_{1}^{r} \rho_{2}^{r}}{\left(\rho_{1}^{r}+s\right)\left(\rho_{2}^{r}+s\right)}}}_{\begin{array}{c}
\text { Divergence } \\
\text { factor }
\end{array}} \underbrace{e^{-j k s}}_{\begin{array}{c}
\text { Phase } \\
\text { factor }
\end{array}}
$$

where $\vec{E}^{i}\left(\vec{Q}_{R}\right)$ is the incident nield at $\vec{Q}_{R}, s$ is distance along the reflected ray from the surface to $\vec{P}, \rho_{1}^{r}$ and $\rho_{2}^{r}$ are the principal radii of curvature of the reflected wave front at $\vec{Q}_{R}, \ddot{R}$ is a dyadic reflection coefficient and $k$ is the wave number in the medium.

While (1) appears relatively simple, the computations involved in its application can become very time consuming. To locate the specular points the surface must be searched for points that result in relative minima or maxima of ray length from source to evaluation point. If the surface is known only at discrete points, a continuous function such as a bicubic spline is fit over each region before searching for a specular point. The radii of curvature of the reflected wave front at specular points is then computed from a formula involving the principal radii of curvature of the surface at $\vec{Q}_{R}$ and of the incident wave front.

Rather than using this approach on terrain surfaces, we have used a method of ray launching proposed by Mittra and Rushdi [1]. This approach was developed for surfaces specified numerically over a grid of points, and traces rays from the known grid points to form triangular ray tubes. Interpolation formulas are developed to determine the factors in (1) from the rays surrounding the evaluation point. The method as described in [1] is limited to convex, perfectly conducting surfaces but is extended here to treat finitely conducting surfaces combining concave and convex regions as occur in hills on a flat ground plane.

\section{The Triangular Ray Tube and Mean Ray}

The ray launching method developed in [1] begins with a surface defined on a grid of points at which the surface elevations and derivatives, or equivalently the unit normal 


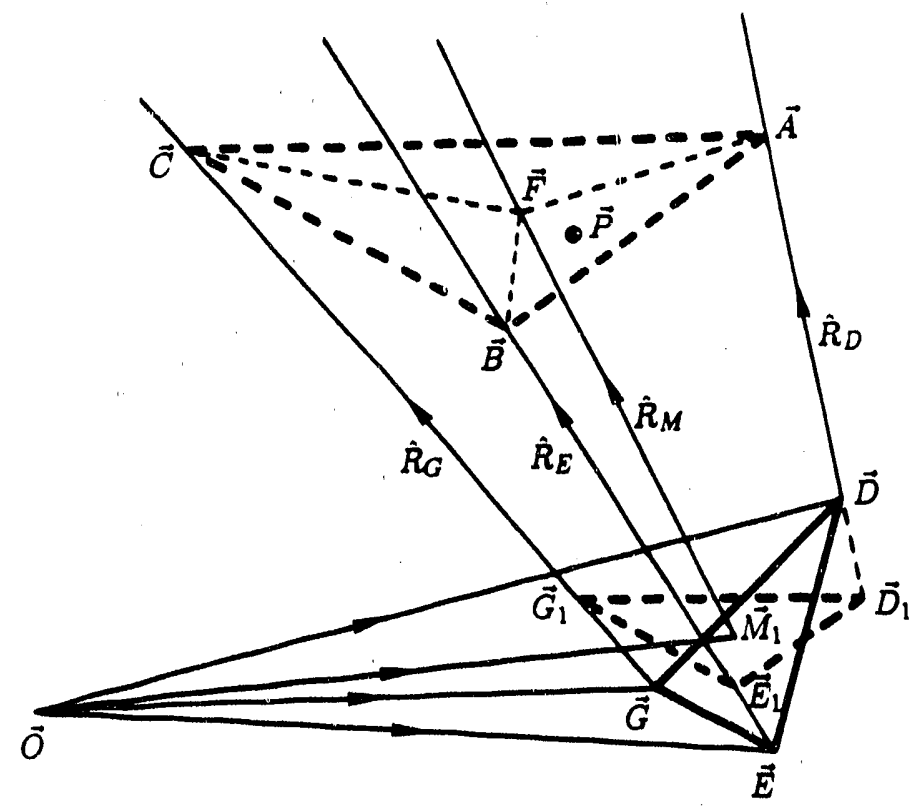

Fig. 1. A triangular ray tube reflecting from points $\vec{D}, \vec{E}$ and $\vec{F}$ on a surface, showing the equiphase triangle $\left(\vec{D}_{1}, \vec{E}_{1}, \vec{G}_{1}\right)$ and the triangle normal to the mean ray $(\vec{A}, \vec{B}, \vec{C})$.

vectors, are known. If the surface elevation is given by $z=f(x, y)$ the unit normal vector is

$$
\hat{n}(x, y)=\frac{\nabla[z-f(x, y)]}{|\nabla[z-f(x, y)]|} .
$$

Rays are launched from the source to groups of three adjacent points on the surface, forming triangular ray tubes as illustrated in Fig. 1. The field evaluation point is then tested to determine whether it is within the particular ray tube and that the ray path is unobstructed. If the ray tube includes the evaluation point then the reflected field, divergence factor and phase factor in (1) are evaluated at the receiver location by interpolation of the surrounding rays.

Rays from the source at $\vec{O}$ to the points $\vec{D}, \vec{E}$ and $\vec{G}$ will be denoted $\vec{S}_{D}=\vec{D}-\vec{O}$, $\vec{S}_{E}=\vec{E}-\vec{O}$ and $\vec{S}_{G}=\vec{G}-\vec{O}$. The reflected r xys are then

$$
\begin{array}{ll}
\vec{R}_{D}=\vec{S}_{D}-2\left(\vec{S}_{D} \cdot \hat{n}_{D}\right) \hat{n}_{D}, & \hat{R}_{D}=\vec{R}_{D} / \mid \vec{R}_{i} \\
\vec{R}_{E}=\vec{S}_{E}-2\left(\vec{S}_{E} \cdot \hat{n}_{E}\right) \hat{n}_{E}, & \hat{R}_{E}=\vec{R}_{E} /\left|\vec{R}_{E}\right| \\
\vec{R}_{G}=\vec{S}_{G}-2\left(\vec{S}_{G} \cdot \hat{n}_{G}\right) \hat{n}_{G}, & \hat{R}_{G}=\vec{R}_{G} /\left|\vec{R}_{G}\right|
\end{array}
$$

where $\hat{n}_{D}, \hat{n}_{E}$ and $\hat{n}_{G}$ are unit normal vectors at $\vec{D}, \vec{E}$ and $\vec{G}$.

A mean ray is associated with the reflected ray tube for use in developing the interpolation formulas. This mean ray is taken to reflect from a surface intersecting the reflected rays at points adjusted to have equal phase from the source. This equiphase triangle then represents an approximation of the reflected wave front. Mittra and Rushdi sort the rays 
so that $\left|\vec{S}_{G}\right| \leq\left|\vec{S}_{E}\right| \leq\left|\vec{S}_{D}\right|$ and locate the equiphase triangle so that the corner on ray $\mathrm{E}$ lies on the reflecting surface at $\vec{E}$. We found better accuracy in the phase interpolation, described later, when ti?e equiphase triangle was adjusted by the mean distance from the source rather than the median. Hence, using the mean distance

$$
S_{M}=\left(\left|\vec{S}_{D}\right|+\left|\vec{S}_{E}\right|+\left|\vec{S}_{G}\right|\right) / 3
$$

the equiphase triangle shown in Fig. 1 is defined as

$$
\begin{aligned}
& \vec{D}_{1}=\vec{D}-\left(\left|\vec{S}_{D}\right|-S_{M}\right) \hat{R}_{D} \\
& \vec{E}_{1}=\vec{E}-\left(\left|\vec{S}_{E}\right|-S_{M}\right) \hat{R}_{E} \\
& \vec{G}_{1}=\vec{G}-\left(\left|\vec{S}_{G}\right|-S_{M}\right) \hat{R}_{G} .
\end{aligned}
$$

The mean ray is then taken to reflect from the centroid of this equiphase triangle at the surface

$$
\vec{M}_{1}=\left(\vec{D}_{1}+\vec{E}_{1}+\vec{G}_{1}\right) / 3
$$

with incident mean ray $\vec{S}_{M}=\vec{M}_{1}-\vec{O}$. The reflected mean ray passes through the centroid of the triangle $\left(\vec{D}_{2}, \vec{E}_{2}, \vec{G}_{2}\right)$ where

$$
\begin{aligned}
& \vec{D}_{2}=\vec{D}_{1}+L \hat{R}_{D} \\
& \vec{E}_{2}=\vec{E}_{1}+L \hat{R}_{E} \\
& \overrightarrow{\vec{G}}_{2}=\vec{G}_{1}+L \hat{R}_{G}
\end{aligned}
$$

and $L$ is an arbitrary distance. Fience the direction of the reflected mean ray is

$$
\hat{R}_{m}=\left(\hat{R}_{D}+\hat{R}_{E}+\hat{R}_{G}\right) /\left|\hat{R}_{D}+\hat{R}_{E}+\hat{R}_{G}\right|
$$

The plane normal to this mean ray is also an approximation to the reflected wave front. It is nearly parallel to the equiphase triangle $\left(\vec{D}_{2}, \vec{E}_{2}, \vec{G}_{2}\right)$, although they do not coincide.

Before going any further in the field evaluation we need to determine whether the evaluation point is contained in a particular triangular ray tube. Mittra and Rushdi make this determination by searching for the mean ray that comes closest to the evaluation point $\vec{P}$. However, when concave surfaces are allowed there can be multiple rays to a given evaluation point, so a search for a minimum distance would have to consider relative minima. A more direct test is whether the evaluation point is contained in a triangle formed by the ray tube.

One possible triangle for testing is that in the plane normal to the mean ray and containing $\vec{P}$. The vertices of this triangle are

$$
\begin{aligned}
& \vec{A}=\vec{D}+\lambda_{a} \hat{R}_{D} \\
& \vec{B}=\vec{E}+\lambda_{b} \hat{R}_{E} \\
& \vec{C}=\vec{G}+\lambda_{c} \hat{R}_{G}
\end{aligned}
$$


where

$$
\begin{aligned}
& \lambda_{a}=\hat{R}_{m} \cdot(\vec{P}-\vec{D}) /\left(\hat{R}_{m} \cdot \hat{R}_{D}\right) \\
& \lambda_{b}=\hat{R}_{m} \cdot(\vec{P}-\vec{E}) /\left(\hat{R}_{m} \cdot \hat{R}_{E}\right) \\
& \lambda_{c}=\hat{R}_{m} \cdot(\vec{P}-\vec{G}) /\left(\hat{R}_{m} \cdot \hat{R}_{G}\right)
\end{aligned}
$$

The point $\vec{P}$ is contained in this triangle if it is included in two angles such as $\mathrm{CAB}$ and $A B C$. Hence a suitable test for inclusion is

$$
\begin{aligned}
& {[(\vec{B}-\vec{A}) \times(\vec{P}-\vec{A})] \cdot[(\vec{C}-\vec{A}) \times(\vec{P}-\vec{A})] \leq 0} \\
& {[(\vec{C}-\vec{B}) \times(\vec{P}-\vec{B})] \cdot[(\vec{A}-\vec{B}) \times(\vec{P}-\vec{B})] \leq 0}
\end{aligned}
$$

and

$$
[(\vec{B}-\vec{A})+(\vec{C}-\vec{A})] \cdot(\vec{P}-\vec{A})>0 .
$$

An alternate test based on areas is used in [1].

A problem with testing with the triangle normal to the mean ray is that the triangles for adjacent ray tubes lie in different planes and do not meet at the edges. Since the reflected rays do not in general converge to a point there may be a small overlap or gap between these triangles as viewed along the mean rays. To avoid the possibility of gaps in the surface, our present code performs an initial test on triangles formed on a horizontal plane containing point $\vec{P}$. Vertices of the horizontal triangle in a ray tube are

$$
\begin{aligned}
& \vec{D}_{H}=\vec{D}+h_{D} \hat{R}_{D} \\
& \vec{E}_{H}=\vec{E}+h_{E} \hat{R}_{E} \\
& \vec{G}_{H}=\vec{G}+h_{G} \hat{R}_{G}
\end{aligned}
$$

where

$$
\begin{aligned}
& h_{D}=(\vec{P}-\vec{D}) \cdot \hat{z} /\left(\hat{R}_{D} \cdot \hat{z}\right) \\
& h_{E}=(\vec{P}-\vec{E}) \cdot \hat{z} /\left(\hat{R}_{E} \cdot \hat{z}\right) \\
& h_{G}=(\vec{P}-\vec{G}) \cdot \hat{z} /\left(\hat{R}_{G} \cdot \hat{z}\right) .
\end{aligned}
$$

The test for $\vec{P}$ included in this triangle can use (7) through (9) with the $z$ components eliminated. Any other continuous surface, such as a hemisphere, could be used for testing, but the horizontal plane is easy to use. When the test on a horizontal plane fails because the reflected rays are nearly horizontal, the triangle normal to the mean ray was used.

\section{Evaluation of the Divergence Factor}

The ray divergence factor in (1) depends on the principal radii of curvature of the reflected wave front at the surface. To determine the radii of curvature from the triangular ray tube would require a difficult interpolation procedure. An easier method is to derive the divergence factor from the square root of the ratio of the cross sectional areas of the 
ray tube at the surface and at the evaluation point $\vec{P}$. This was done in [1] by evaluating the area of the equiphase triangle, defined in (4), for three discrete values of $L$ to obtain a quadratic equation for the area as a function of $L$.

In order to handle concave reflecting surfaces, the caustics along the ray tube must be located to determine whether they fall between the surface and the evaluation point. A problem with using the equiphase triangle for this purpose is that, in the neighborhood of a caustic, the equiphase triangle does not go through zero area. Instead, it gets small and then rotates to lie in the plane of the mean ray. The triangle normal to the mean ray cannot do this, and in general has two points of zero area that can be used as approximations for the location of caustics. Hence the divergence factor was determined from the area of the triangle normal to the mean ray.

The triangle normal to the mean ray at point $\vec{p}=\vec{M}_{1}+\ell \hat{R}_{m}$ has vertices

$$
\begin{aligned}
& \vec{D}_{n}=\vec{D}+\ell_{D} \hat{R}_{D} \\
& \vec{E}_{n}=\vec{E}+\ell_{E} \hat{R}_{E} \\
& \vec{G}_{n}=\vec{G}+\ell_{G} \hat{R}_{G}
\end{aligned}
$$

where

$$
\begin{aligned}
& \ell_{D}=\hat{R}_{m} \cdot(\vec{p}-\vec{D}) /\left(\hat{R}_{m} \cdot \hat{R}_{D}\right) \\
& \ell_{E}=\hat{R}_{m} \cdot(\vec{p}-\vec{E}) /\left(\hat{R}_{m} \cdot \hat{R}_{E}\right) \\
& \ell_{G}=\hat{R}_{m} \cdot(\vec{p}-\vec{G}) /\left(\hat{R}_{m} \cdot \hat{R}_{G}\right) .
\end{aligned}
$$

Substituting (11) and the expression for $\vec{p}$ into (10) yields

$$
\begin{aligned}
& \vec{D}_{n}=\vec{D}+\frac{\hat{R}_{m} \cdot\left(\vec{M}_{1}-\vec{D}\right)}{\hat{R}_{m} \cdot \vec{R}_{D}} \hat{R}_{D}+\frac{\hat{R}_{D}}{\hat{R}_{m} \cdot \hat{R}_{D}} \ell \\
& \vec{E}_{n}=\vec{E}+\frac{\hat{R}_{m} \cdot\left(\vec{M}_{1}-\vec{E}\right)}{\hat{R}_{m} \cdot \vec{R}_{E}} \hat{R}_{E}+\frac{\hat{R}_{E}}{\hat{R}_{m} \cdot \hat{R}_{E}} \ell \\
& \vec{G}_{n}=\vec{G}+\frac{\hat{R}_{m} \cdot\left(\vec{M}_{1}-\vec{G}\right)}{\hat{R}_{m} \cdot \vec{R}_{G}} \hat{R}_{G}+\frac{\hat{R}_{G}}{\hat{.}_{m} \cdot \hat{R}_{G}} \ell
\end{aligned}
$$

The area of the triangle is

$$
\begin{aligned}
A(\ell) & =\frac{1}{2}\left|\hat{R}_{m} \cdot\left(\vec{E}_{n}-\vec{D}_{n}\right) \times\left(\vec{G}_{n}-\vec{D}_{n}\right)\right| \\
& =\frac{1}{2}\left|\hat{R}_{m} \cdot\left\{\left(\vec{P}_{1} \times \vec{P}_{2}\right) \ell^{2}+\left[\left(\vec{P}_{1} \times \vec{Q}_{2}\right)+\left(\vec{Q}_{1} \times \vec{P}_{2}\right)\right] \ell+\left(\vec{Q}_{1} \times \vec{Q}_{2}\right)\right\}\right|
\end{aligned}
$$


where

$$
\begin{aligned}
& \vec{P}_{1}=\frac{\hat{R}_{E}}{\hat{R}_{m} \cdot \hat{R}_{E}}-\frac{\hat{R}_{D}}{\hat{R}_{m} \cdot \hat{R}_{D}} \\
& \vec{P}_{2}=\frac{\hat{R}_{G}}{\hat{R}_{m} \cdot \hat{R}_{G}}-\frac{\hat{R}_{D}}{\hat{R}_{m} \cdot \hat{R}_{D}} \\
& \vec{Q}_{1}=\vec{E}-\vec{D}+\frac{\hat{R}_{m} \cdot\left(\vec{M}_{1}-\vec{E}\right)}{\hat{R}_{m} \cdot \vec{R}_{E}} \hat{R}_{E}-\frac{\hat{R}_{m} \cdot\left(\vec{M}_{1}-\vec{D}\right)}{\hat{R}_{m} \cdot \vec{R}_{D}} \hat{R}_{D} \\
& \vec{Q}_{2}=\vec{G}-\vec{E}+\frac{\hat{R}_{m} \cdot\left(\vec{M}_{1}-\vec{G}\right)}{\hat{R}_{m} \cdot \vec{R}_{G}} \hat{R}_{G}-\frac{\hat{R}_{m} \cdot\left(\vec{M}_{1}-\vec{D}\right)}{\hat{R}_{m} \cdot \vec{R}_{D}} \hat{R}_{D}
\end{aligned}
$$

Thus the area is a quadratic function of the distance $\ell$

$$
A(\ell)=\left|q \ell^{2}+r \ell+s\right|
$$

where

$$
\begin{aligned}
& q=\vec{P}_{2} \cdot\left(\hat{R}_{m} \times \vec{P}_{1}\right) \\
& r=\vec{Q}_{2} \cdot\left(\hat{R}_{m} \times \vec{P}_{1}\right)+\vec{P}_{2} \cdot\left(\hat{R}_{m} \times \vec{Q}_{1}\right) \\
& s=\vec{Q}_{2} \cdot\left(\hat{R}_{m} \times \vec{Q}_{1}\right) .
\end{aligned}
$$

In (12) through (14) the triple products have been rearranged so that only two cross products need to be evaluated.

Roots of the equation

$$
q \ell_{c}^{2}+r \ell_{c}+s=0
$$

represent caustics in the ray tube, where one vertex of the triangle crosses the line through the other two, so that the area goes to zero. The GO model is not valid at the caustics, but these points are usually apparent due to large, anomalous field values when the caustic coincides with the evaluation point. The phase of the field advances by $\pi / 2$ as the observer passes through a caustic, as is evident from the negative argument of the square root in (1). Hence the field must be multiplied by $j=\sqrt{-1}$ for each root of (15) for which

$$
0<\ell_{c}<\lambda_{p}
$$

where

$$
\lambda_{p}=\hat{R}_{m} \cdot\left(\vec{P}-\vec{M}_{1}\right) .
$$

Thus if $N_{c}$ is the number of roots of (15) satisfying (16) the divergence factor, including phase shifts due to coustics, is

$$
D_{f}(\vec{P})=j^{N_{e}} \sqrt{A(0) / A\left(\lambda_{p}\right)} .
$$

\section{Phase Interpolation}

To evaluate the phase of the field at the evaluation point $\vec{P}$ a quadratic interpolation formula was applied as done in [1]. The phase is interpolated in the plane normal to the 
mean ray, defined in (5), with the quadratic function fit to the phase at $\vec{A}, \vec{B}, \vec{C}$ and $\vec{F}$. $\vec{F}$ is the intersection of the mean ray with the normal triangle containing $\vec{P}$

$$
\vec{F}=\vec{M}_{1}+\lambda_{p} \hat{R}_{m}
$$

where

$$
\lambda_{p}=\hat{R}_{m} \cdot\left(\vec{P}-\vec{M}_{1}\right)
$$

The value of including $\vec{F}$ seems uncertain. The phase is known ex ctly at $\vec{A}, \vec{B}$ and $\vec{C}$ since the surface is known at the points where those rays reflect. However, the phase at $\vec{F}$ depends on the somewhat arbitrary location of the equiphase triangle defined in (2). The use of the mean distance from the source in locating the equiphase triangle gave Detter results than the median as used in [1], particularly when the incident ray arrived normal to the side of the triangle so that the median became an extreme value. A more accurate result might be obtained by fitting a continuous function to the surface and finding the intersection of the mekis ray with this surface. This interpolated reflection point could then be used in computing an accurate phase along the mean ray. However, this more complex procedure was not tried.

Using the roordinates $x_{1}$ and $x_{2}$ defined along the orthogonal vectors $\hat{x}_{1}$ and $\hat{x}_{2}$, the quadratic interpolation fits the function

$$
\phi\left(x_{1}, x_{2}\right)=\frac{k}{2}\left[Q_{11} x_{1}^{2}+\left(Q_{12}+Q_{21}\right)_{2} x_{2}+Q_{22} x_{2}^{2}\right]
$$

to the phase at $\vec{A}, \vec{B}$ and $\vec{C}$ relative to that at $\vec{F}$,

$$
\begin{aligned}
& \Phi_{A}=k\left[\lambda_{a}+\left|\vec{S}_{D}\right|-\left(\lambda_{p}+\left|\vec{S}_{M}\right|\right)\right] \\
& \Phi_{B}=k\left[\lambda_{b}+\left|\vec{S}_{E}\right|-\left(\lambda_{p}+\left|\vec{S}_{M}\right|\right)\right] \\
& \Phi_{C}=k\left[\lambda_{c}+\left|\vec{S}_{G}\right|-\left(\lambda_{p}+\left|\vec{S}_{M}\right|\right)\right]
\end{aligned}
$$

where $\lambda_{a}, \lambda_{b}$ and $\lambda_{c}$ are from (6). The interpolation constants are determined by solving the equations

$$
\begin{aligned}
& \Phi_{A}=\frac{k}{2}\left[Q_{11} x_{1 a}^{2}+\left(Q_{12}+Q_{21}\right) x_{1 a} x_{2 a}+Q_{2 c}\right] \\
& \Phi_{B}=\frac{k}{2}\left[Q_{11} x_{1 b}^{2}+\left(Q_{12}+Q_{21}\right) x_{1 b} x_{2 b}+Q_{22} x_{2 b}^{2}\right] \\
& \Phi_{C}=\frac{k}{2}\left[Q_{11} x_{1 c}^{2}+\left(Q_{12}+Q_{21}\right) x_{1 c} x_{2 c}+Q_{22} x_{2 c}^{2}\right]
\end{aligned}
$$

where

$$
\begin{aligned}
& x_{i a}=(\vec{A}-\vec{F}) \cdot \hat{x}_{i} \\
& x_{i b}=(\vec{B}-\vec{F}) \cdot \hat{x}_{i} \\
& x_{i c}=(\vec{C}-\vec{F}) \cdot \hat{x}_{i}
\end{aligned}
$$

for $i=1,2$. As done in [1], the solution is simplified by choosing

$$
\hat{x}_{1}=(\vec{P}-\vec{F}) /|\vec{P}-\vec{F}|
$$


and

$$
\hat{x}_{2}=\hat{R}_{m} \times \hat{x}_{1} .
$$

Then the interpolated phase at $\vec{P}$ is given by

$$
\phi(|\vec{P}-\vec{F}|, 0)=\frac{k}{2} Q_{11}|\vec{P}-\vec{F}|^{2}
$$

so that only $Q_{11}$ must be evaluated. The solution of the equations (18) yields

$$
\frac{k}{2} Q_{11}=\frac{\Phi_{A} \Delta_{1}+\Phi_{B} \Delta_{2}+\Phi_{C} \Delta_{3}}{\left(x_{1 a} x_{2 b}-x_{1 b} x_{2 a}\right)\left(x_{1 b} x_{2 c}-x_{1 c} x_{2 b}\right)\left(x_{1 c} x_{2 a}-x_{1 a} x_{2 c}\right)}
$$

where

$$
\begin{aligned}
& \Delta_{1}=x_{2 b}^{2} x_{1 c} x_{2 c}-x_{2 c}^{2} x_{1 b} x_{2 b} \\
& \Delta_{2}=x_{2 c}^{2} x_{1 a} x_{2 a}-x_{2 a}^{2} x_{1 c} x_{2 c} \\
& \Delta_{3}=x_{2 a}^{2} x_{1 b} x_{2 b}-x_{2 b}^{2} x_{1 a} x_{2 a} .
\end{aligned}
$$

The phase at $\vec{P}$ is then approximated as

$$
\Phi(\vec{P})=k\left(\lambda_{p}+\left|\vec{S}_{M}\right|+\frac{1}{2} Q_{11}|\vec{P}-\vec{F}|^{2}\right) .
$$

\section{Interpolation of the Reflected Field}

The reflected field for a ray that would reach point $\vec{P}$ is approximated by linear interpolation of the reflected fields for rays to points $\vec{D}, \vec{E}$ and $\vec{G}$. For a perfectly conducting surface only the change in vector direction from incident to reflected field must be determined. For finitely conducting ground the reflected field is obtained from the plane wave reflection coefficients, since the wave front can be treated as essentially plane at the surface if the source is distant. If the source is near the terrain surface but the receiver is distant the plane-wave reflection coefficient can still be used by applying reciprocity.

The incident wave is decomposed into TE and TM components relative to the plane of incidence, defined by the incident ray $\hat{s}$ and the normal to the surface $\hat{n}$. The unit vector normal to the plane of incidence is

$$
\hat{p}=(\hat{s} \times \hat{n}) /|\hat{s} \times \hat{n}|
$$

and the incidence angle is

$$
\theta_{i}=\cos ^{-1}(-\hat{n} \cdot \hat{s}) .
$$

From Snell's law, the angle $\theta_{t}$ of a wave transmitted into the medium is

$$
\cos \theta_{t}=\left[1-\frac{\epsilon_{0}}{\epsilon_{1}}\left(1-\cos ^{2} \theta_{i}\right)\right]^{1 / 2}
$$

where $\epsilon_{0}$ and $\epsilon_{1}$ are the permittivities of free space and ground, respectively. 
The reflection coefficients for TE and TM polarizations can be computed in terms of surface impedance and admittance as

$$
\begin{gathered}
R^{\mathrm{TE}}=\frac{Y_{0}^{\mathrm{TE}}-Y_{s}^{\mathrm{TE}}}{Y_{0}^{\mathrm{TE}}+Y_{s}^{\mathrm{TE}}} \\
R^{\mathrm{TM}}=-\frac{Z_{0}^{\mathrm{TM}}-Z_{s}^{\mathrm{TM}}}{Z_{0}^{\mathrm{TM}}+Z_{s}^{\mathrm{TM}}}
\end{gathered}
$$

where

$$
\begin{aligned}
Y_{0}^{\mathrm{TE}}=\eta_{0}^{-1} \cos \theta_{i}, & Y_{s}^{\mathrm{TE}}=\eta_{1}^{-1} \cos \theta_{t} \\
Z_{0}^{\mathrm{TM}}=\eta_{0} \cos \theta_{i}, & Z_{s}^{\mathrm{TM}}=\eta_{1} \cos \theta_{t}
\end{aligned}
$$

with $\eta_{0}=\sqrt{\mu_{0} / \epsilon_{0}}$ and $\eta_{1}=\sqrt{\mu_{0} / \epsilon_{1}}$. For a perfectly conducting ground, $R^{\mathrm{TE}}=R^{\mathrm{TM}}=$ -1 .

Decomposing the incident electric field $\vec{E}^{i}$ as

$$
\begin{aligned}
& \vec{E}_{\mathrm{TE}}^{i}=\left(\vec{E}^{i} \cdot \hat{p}\right) \hat{p} \\
& \vec{E}_{\mathrm{TM}}^{i}=\vec{E}^{i}-\left(\vec{E}^{i} \cdot \hat{p}\right) \hat{p}
\end{aligned}
$$

the reflected field is obtained as

$$
\begin{aligned}
& \vec{E}_{T E}^{r}=\vec{E}_{\mathrm{TE}}^{\mathrm{i}} R^{\mathrm{TE}} \\
& \vec{E}_{\mathrm{TM}}^{\mathrm{r}}=\left[\vec{E}_{\mathrm{TM}}^{\mathrm{i}}-2\left(\vec{E}^{i} \cdot \hat{n}\right) \hat{n}\right] R^{\mathrm{TM}} .
\end{aligned}
$$

The total reflected electric field at the surface for an incident field $\vec{E}^{i}$ is then

$$
\vec{E}^{r}=\overline{\bar{R}}\left[\vec{E}^{i}\right]=\left[\vec{E}^{i}-2\left(\vec{E}^{i} \cdot \hat{n}\right) \hat{n}\right] R^{\mathrm{TM}}+\hat{p}\left(\vec{E}^{i} \cdot \hat{p}\right)\left(R^{\mathrm{T} E}-R^{\mathrm{TM}}\right) .
$$

Linear interpolation was used to obtain the magnitude and vector direction of the reflected field at the surface on the ray to $\vec{P}$ from the rays to points $\vec{D}, \vec{E}$ and $\vec{G}$. The interpolation function

$$
\vec{E}^{r}\left(\xi_{1}, \xi_{2}\right)=\vec{q}_{1} \xi_{1}+\vec{q}_{2} \xi_{2}+\vec{q}_{3}
$$

is matched to the reflected field at $\vec{D}, \vec{E}$ and $\vec{G}$ to determine $\overrightarrow{q_{1}}, \overrightarrow{q_{2}}$ and $\vec{q}_{3}$. The result can be simplified by defining the coordinates in the plane of $\vec{A}, \vec{B}$ and $\vec{C}$ with origin at $\vec{A}$ and

$$
\begin{aligned}
& \hat{\xi}_{1}=(\vec{P}-\vec{A}) /|\vec{P}-\vec{A}| \\
& \hat{\xi}_{2}=\hat{R}_{m} \times \hat{\xi}_{1} .
\end{aligned}
$$

The equations to be solved for $\overrightarrow{q_{1}}, \overrightarrow{q_{2}}$ and $\overrightarrow{q_{3}}$ are then

$$
\begin{aligned}
\overrightarrow{q_{3}} & =\vec{E}_{D}^{r} \\
\vec{q}_{1} \xi_{1 b}+\vec{q}_{2} \xi_{2 b}+\vec{q}_{3} & =\vec{E}_{E}^{r} \\
\vec{q}_{1} \xi_{1 c}+\vec{q}_{2} \xi_{2 c}+\vec{q}_{3} & =\vec{E}_{G}^{r}
\end{aligned}
$$


where

$$
\begin{aligned}
& \xi_{i b}=(\vec{B}-\vec{A}) \cdot \hat{\xi}_{i} \\
& \xi_{i c}=(\vec{C}-\vec{A}) \cdot \hat{\xi}_{i}
\end{aligned}
$$

for $i=1,2$ and

$$
\begin{aligned}
& \vec{E}_{D}^{r}=\overline{\bar{\Re}}\left[\vec{E}^{i}(\vec{D})\right] / e^{-j k\left|\vec{S}_{D}\right|} \\
& \vec{E}_{E}^{r}=\overline{\bar{\Re}}\left[\vec{E}^{i}(\vec{E})\right] / e^{-j k\left|\vec{S}_{E}\right|} \\
& \vec{E}_{G}^{r}=\overline{\bar{\Re}}\left[\vec{E}^{i}(\vec{G})\right] / e^{-j k\left|\vec{S}_{G}\right|} .
\end{aligned}
$$

$\vec{E}^{i}(\vec{R})$ is the incidenc field at $\vec{R}$ and $\vec{\Re}$ is defined in (20). The phase lag from the origin has been removed since it is included in the phase interpolation in (19). The solution of (21) yields

$$
\begin{aligned}
& \vec{q}_{1}=\frac{\xi_{2 c}\left(\vec{E}_{E}^{r}-\vec{E}_{D}^{r}\right)}{\xi_{1 b} \xi_{2 c}-\xi_{1 c} \xi_{2 b}}-\frac{\xi_{2 b}\left(\vec{E}_{G}^{r}-\vec{E}_{D}^{r}\right)}{\xi_{1 b} \xi_{2 c}-\xi_{1 c} \xi_{2 b}} \\
& \vec{q}_{2}=\frac{\xi_{1 b}\left(\vec{E}_{G}^{r}-\vec{E}_{D}^{r}\right)}{\xi_{1 b} \xi_{2 c}-\xi_{1 c} \xi_{2 b}}-\frac{\xi_{1 c}\left(\vec{E}_{E}^{r}-\vec{E}_{D}^{r}\right)}{\xi_{1 b} \xi_{2 c}-\xi_{1 c} \xi_{2 b}}
\end{aligned}
$$

Since $\xi_{2}=0$ at $\vec{P}$ the reflected field at the surface on the ray to $\vec{P}$ can be approximated as

$$
\vec{E}^{r}(\vec{P})=\vec{E}^{r}\left(\xi_{1 p}, 0\right)=\vec{E}_{D}^{r}+\xi_{1 p}\left[\frac{\xi_{2 c}\left(\vec{E}_{E}^{r}-\vec{E}_{D}^{r}\right)}{\xi_{1 b} \xi_{2 c}-\xi_{1 c} \xi_{2 b}}-\frac{\xi_{2 b}\left(\vec{E}_{G}^{r}-\vec{E}_{D}^{r}\right)}{\xi_{1 b} \xi_{2 c}-\xi_{1 c} \xi_{2 b}}\right]
$$

where $\xi_{1 p}=(\vec{P}-\vec{A}) \cdot \hat{\xi}_{1}$.

\section{The GO Field Evaluation}

In implementing this ray-launching method in a computer code, the surface is defined on a rectangular grid of points at which elevations and unit normal vectors are specified. The surface is scanned for each field evaluation point. When a triangular ray tube is found that captures the evaluation point $\vec{P}$, the incident and reflected rays are tested for blockage by other parts of the terrain. If the ray is unobstructed, the divergence factor, phase and reflected field are evaluated from equations (17), (19) and (22). The field associated with the ray is then evaluated as

$$
\vec{E}^{R}(\vec{P}) \approx \vec{E}^{r}(\vec{P}) D_{f}(\vec{P}) e^{-j \Phi(\vec{P})}
$$

The contributions of all rays that reach $\vec{P}$ are added to the direct ray from the source and any rays that reflect from flat ground outside of the grid region, if these rays are unobstructed. The computation time for multiple evaluation points could be reduced by storing data for the reflected rays or evaluating contributions to all field points in a single scan of the terrain. This was not done to keep the code simple. 


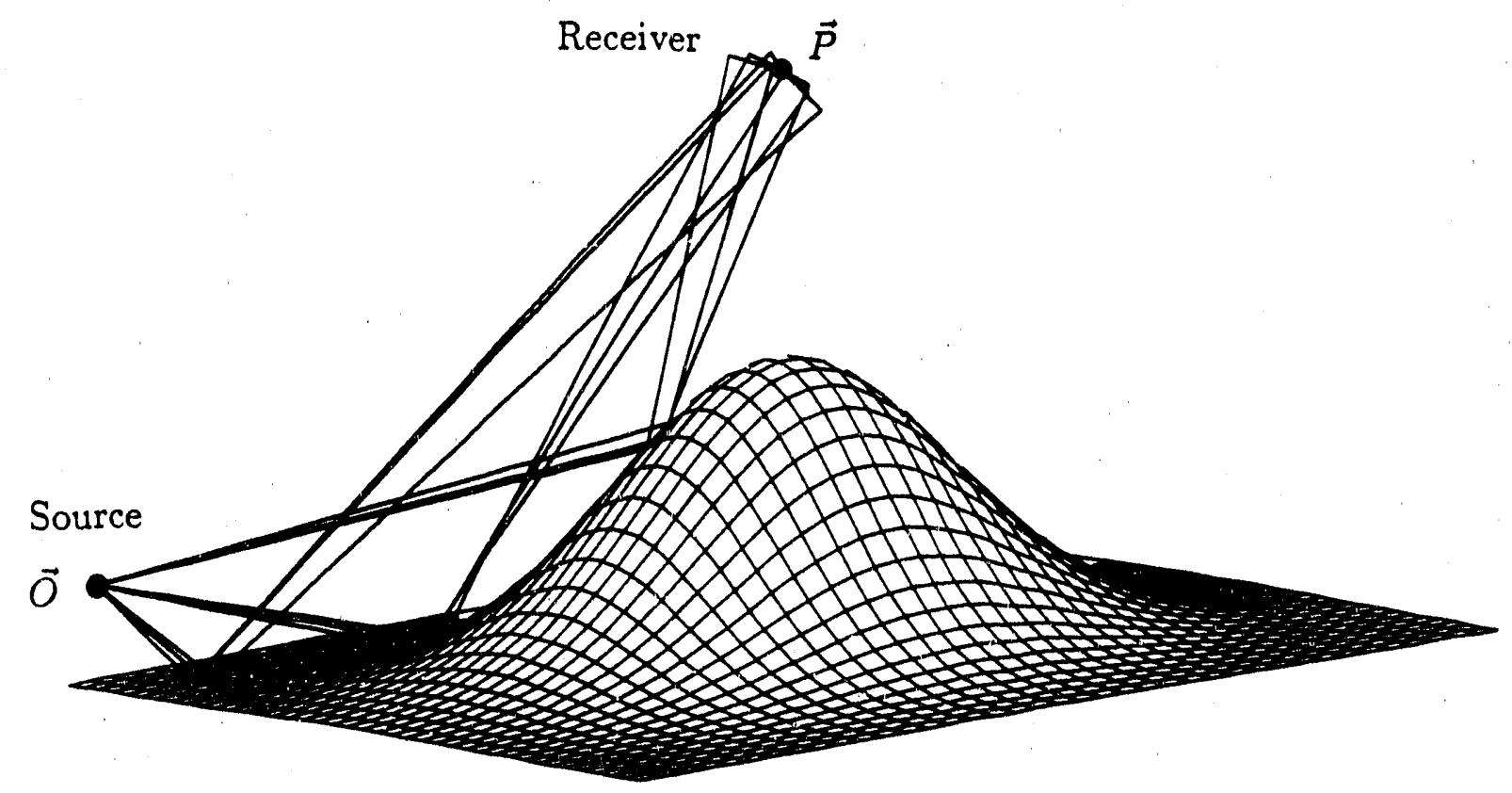

Fig. 2. Triangular ray tubes reflected from a Gaussian Hill.

The triangular ray tubes reflecting from a single Gaussian hill are illustrated in Fig. 2. A caustic, due to focusing of the ray in the vertical plane, can be seen in the ray tube reflecting from the concave surface near the base of the hill. As the evaluation point is moved in a horizontal plane toward the source the two rays reflected from the hill approach each other as they reflect from regions with greater slope. When they converge a caustic occurs at the evaluation point, and the GO field evaluation becomes invalid. As the evaluation point is moved horizontally away from the source another caustic occurs when the lower two rays merge, if they are not first blocked by the peak.

In the present code, only singly reflected rays are considered. However, when the source is located near the surface of a flat ground the direct ray and ray reflected under the source are traced as a single ray through the terrain grid and to the evaluation poirit. The code should be extended to trace at least doubly reflected rays so that the source could be located at any height.

\section{Results from the GO Model for Terrain}

The GO model was tested for scattering from a two dimensional Gaussian ridge to compare with results published by Berry [5] who solved a Volterra integral equation. The solution of this integral equation satisfies a scalar wave equation, so depolarization is neglected. The equation is limited to two dimensional terrain with features constant in the direction transverse to the propagation path. The equation solved by Berry was derived by Hufford [26] using the free-space field as an elementary function. The numerical solution of this equation is less stable than with the equation derived later by Ott [6, 7] using a 
modified Sommerfeld attenuation function. However, an accurate solution of Hufford's equation will show the effects of surface wave and diffraction that are neglected in the GO model.

Berry presented results for a Gaussian ridge defined by

$$
h(x)=H \exp \left[-9\left(\frac{x-b}{w}\right)^{2}\right]
$$

so that the ridge has height $H$, is centered at $b$ and $w$ is the width at about $H / 10$. A vertically polarized source was located at the origin with $H=1 \mathrm{~km}, b=5 \mathrm{~km}$ and $w=3$ $\mathrm{km}$. The ground constants were $\epsilon_{r}=10$ and $\sigma=0.01 \mathrm{~S} / \mathrm{m}$. Berry's results for the vertical component of radiated electric field normalized by the field for a flat, perfectly conducting ground are shown in Fig. 3. An interference pattern is formed above the ridge, with contours approaching a radial direction from the source. Berry shows that the field on the ground behind the ridge, out to $10 \mathrm{~km}$, is dominated by the surface wave at $1 \mathrm{MHz}$, while diffracted field from the ridge top prevails at $10 \mathrm{MHz}$.

Results from the GO model, in Fig. 4, are in general agreement with Berry's solution in the illuminated region but demonstrate some of the limitations of GO. The standing wave pattern above the ridge in Fig. 4 ends abruptly at a caustic surface, while Berry's solution shows the pattern extending to the left of this line. The caustic occurs when the two reflected rays from the ridge, shown in Fig. 2, merge into one. The ideal GO model would predict an infinite field at the caustic, although the numerical solution produces a large, random resuit. In either case the result is not valid. To the left of the caustic, GO shows no effect of the ridge. Also, the perturbation of the field predicted by GO appears to be somewhat smaller than that shown by Berry. There are only small peaks along the caustic exceeding 1.4, while Berry shows significant contours at this level. The weaker scattered field may be the result of a smaller incident field on the ridge in the GO model, as a result of neglecting the surface wave. The lack of surface wave would be less significant at higher frequencies or if the source were moved closer to the ridge. The GO result shows zero field in the shadow of the ridge, while Berry's solution gives a valid prediction in this region.

At $10 \mathrm{MHz}$ the $\mathrm{GO}$ model generates a very complex interference pattern, as would be expected with the $30 \mathrm{~m}$ wavelength. It appears possible that Berry did not fully resolve this pattern. The GO pattern also is not accurately resolved, since an increment of $25 \mathrm{~m}$ was used in distance and height. However, the results from Berry and GO are in reasonable agreement in amplitude. The maximum values in the GO result are about 0.88 near the caustic. The smaller amplitudes at $10 \mathrm{MHz}$ are the result of the greater surface impedance of the ground.

Contour plots of the normalized vertical electric field for a perfectly conducting ridge are shown in Fig. 5. Perieitly conducting ground is of interest due to the possibility of treating real ground as perfectly conducting so that it can be modeled with standard GTD techniques. A surface wave is not generated over perfectly conducting ground, so the main 

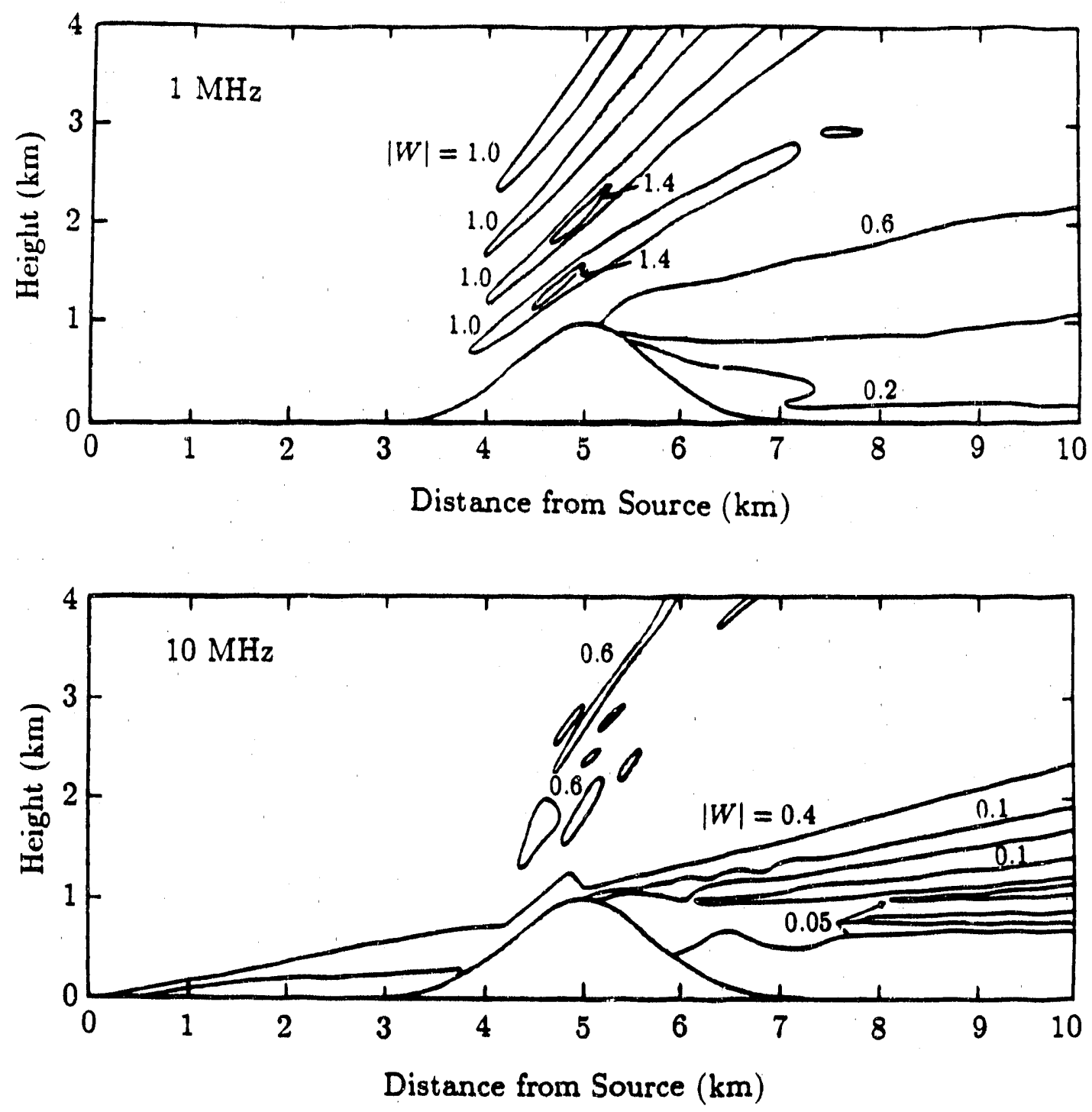

Fig. 3. Contours of normalized vertical electric field over a Gaussian ridge computed by solution of a Volterra integral equation (Source: L. A. Berry, [5], IEEE (C) 1967. Reproduced with permission of IEEE.) Ground parameters are $\epsilon_{g}=10$ and $\sigma=0.01 \mathrm{~S} / \mathrm{m}$.

shortcoming of the GO model is the lack of diffraction or creeping wave at the ridge. For this case the use of perfect conductivity is seen to about double the effect of the ridge, due to the reflection coefficient of 1.0 and a larger field reaching the ridge.

Radiation patterns for the source and Gaussian ridge from Fig. 4 are shown in Fig. 6. The angle $\theta$ is zero overhead and increases toward the ridge. The total $\theta$ component of radiated field was computed and normalized by the same field component for a flat perfectly conducting ground. The pattern was computed at a range of $100 \mathrm{~km}$. The $\theta$ component of field shows a larger perturbation than the vertical component used in the contour plots. Also, the greater resolution with 0.1 degree increments in $\theta$ makies the caustic more evident. Radiation patterns for a perfectly conducting ridge are shown. in Fig. 7. The conductivity is seen to have a substantial effect on the field. 

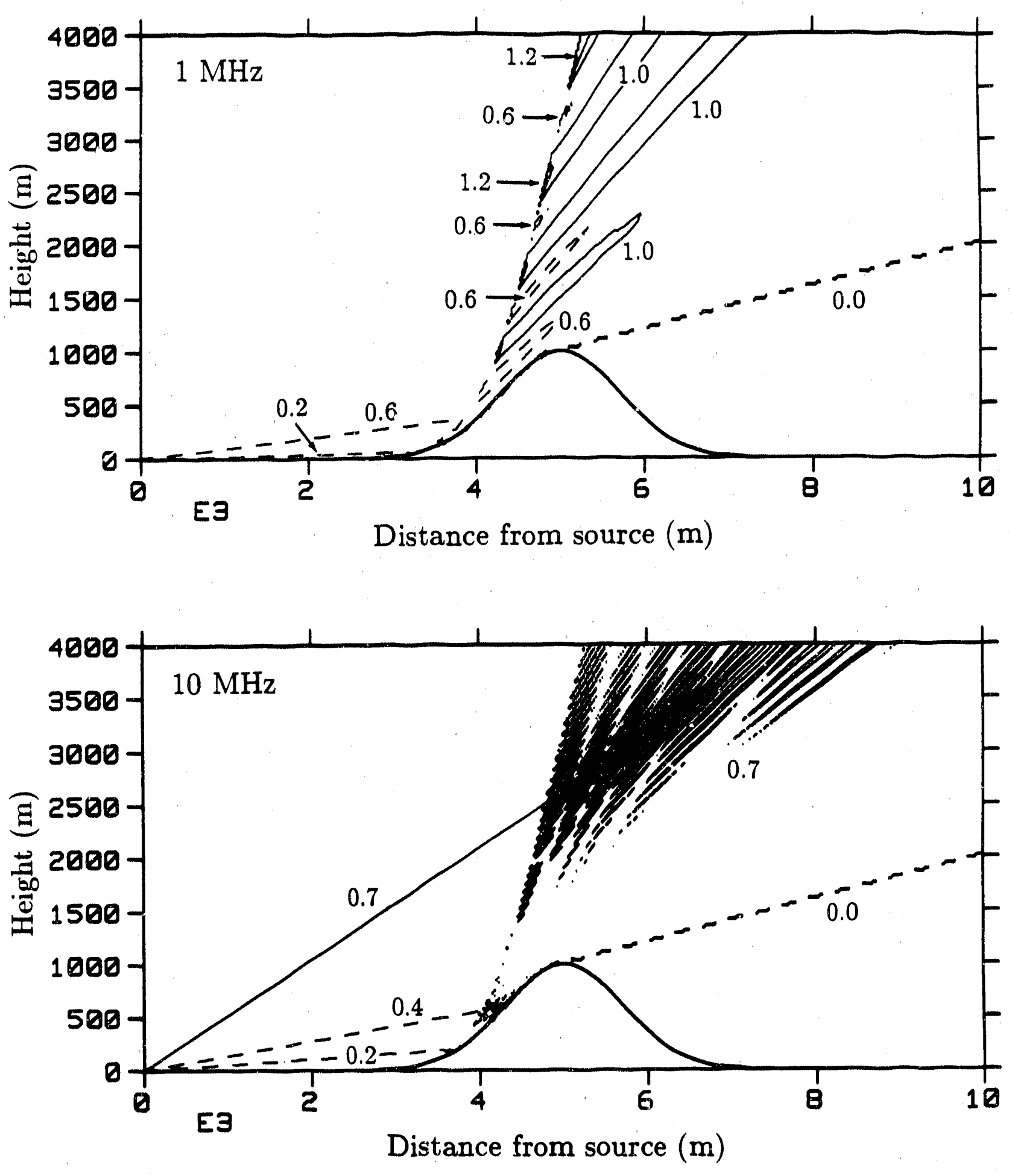

Fig. 4. Contours of normalized vertical electric field over a Gaussian ridge computed by geometrical optics. Ground parameters are $\epsilon_{g}=10$ and $\sigma=0.01 \mathrm{~S} / \mathrm{m}$. The field component $E_{z}$ is normalized to that for a flat, perfectly conducting ground plane. 

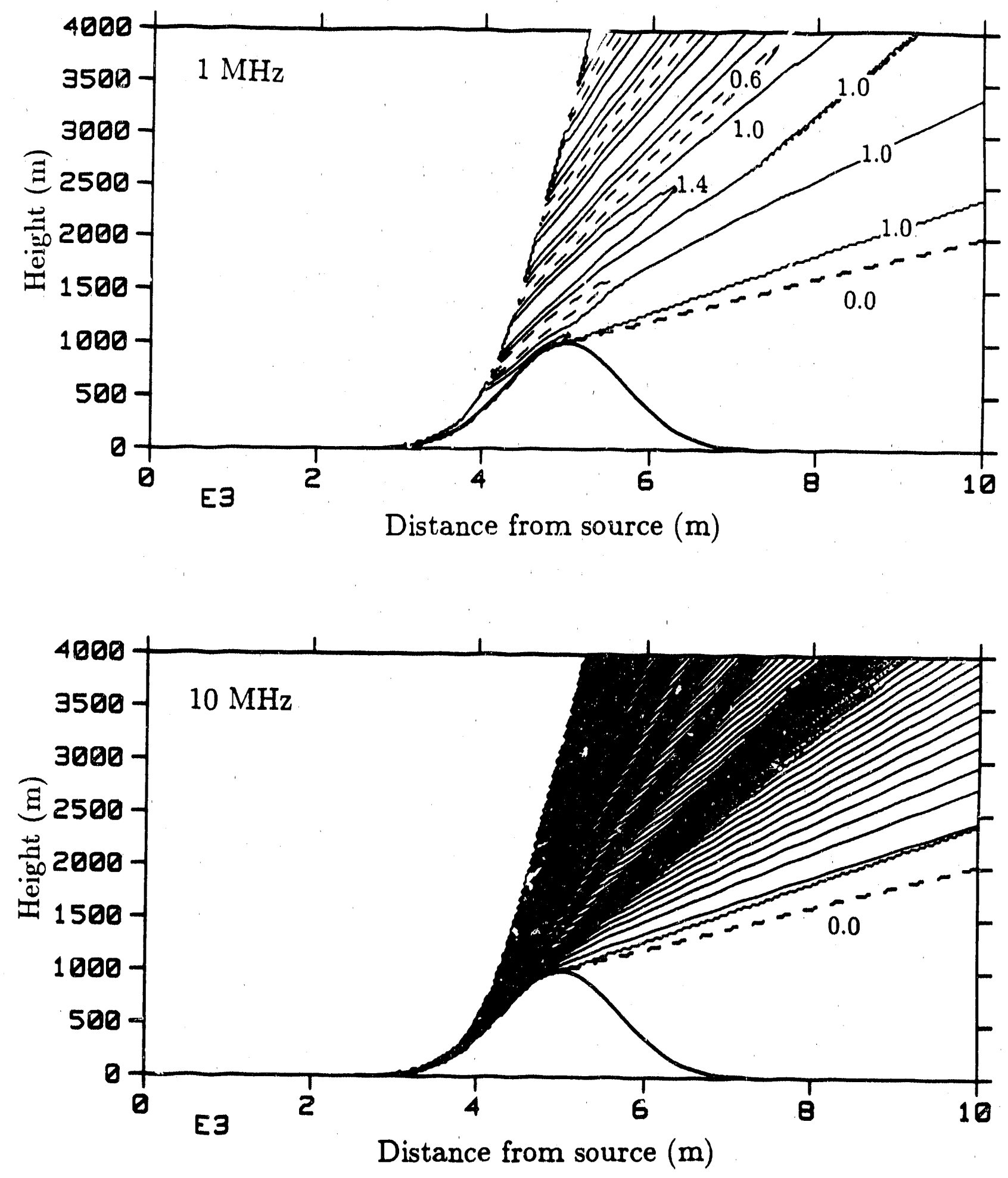

Fig. 5. Contoury of normalized vertical electric field over a Gaussian ridge computed by geometrical optics for perfectly conducting ground. Contour levels are $0.2,0.6,1.0$ and 1.4 

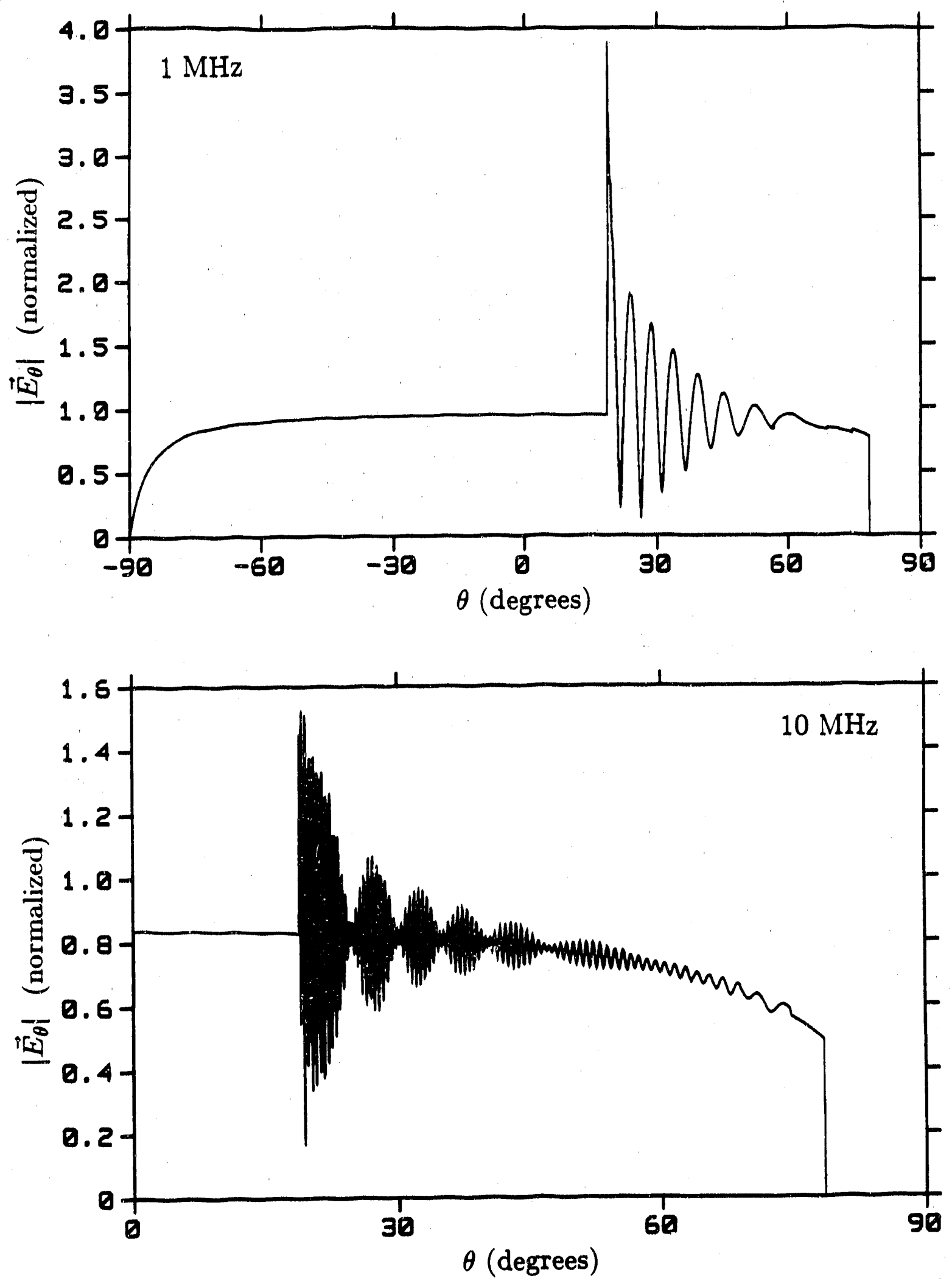

Fig. 6. Radiation pattern for normalized $E_{0}$ over the Gaussian ridge in Fig. 4 with $\epsilon_{g}=10$ and $\sigma=0.01$. $\mathrm{S} / \mathrm{m}$. The field is normalized to $E_{0}$ for a tat, perfectly conducting ground plane. 

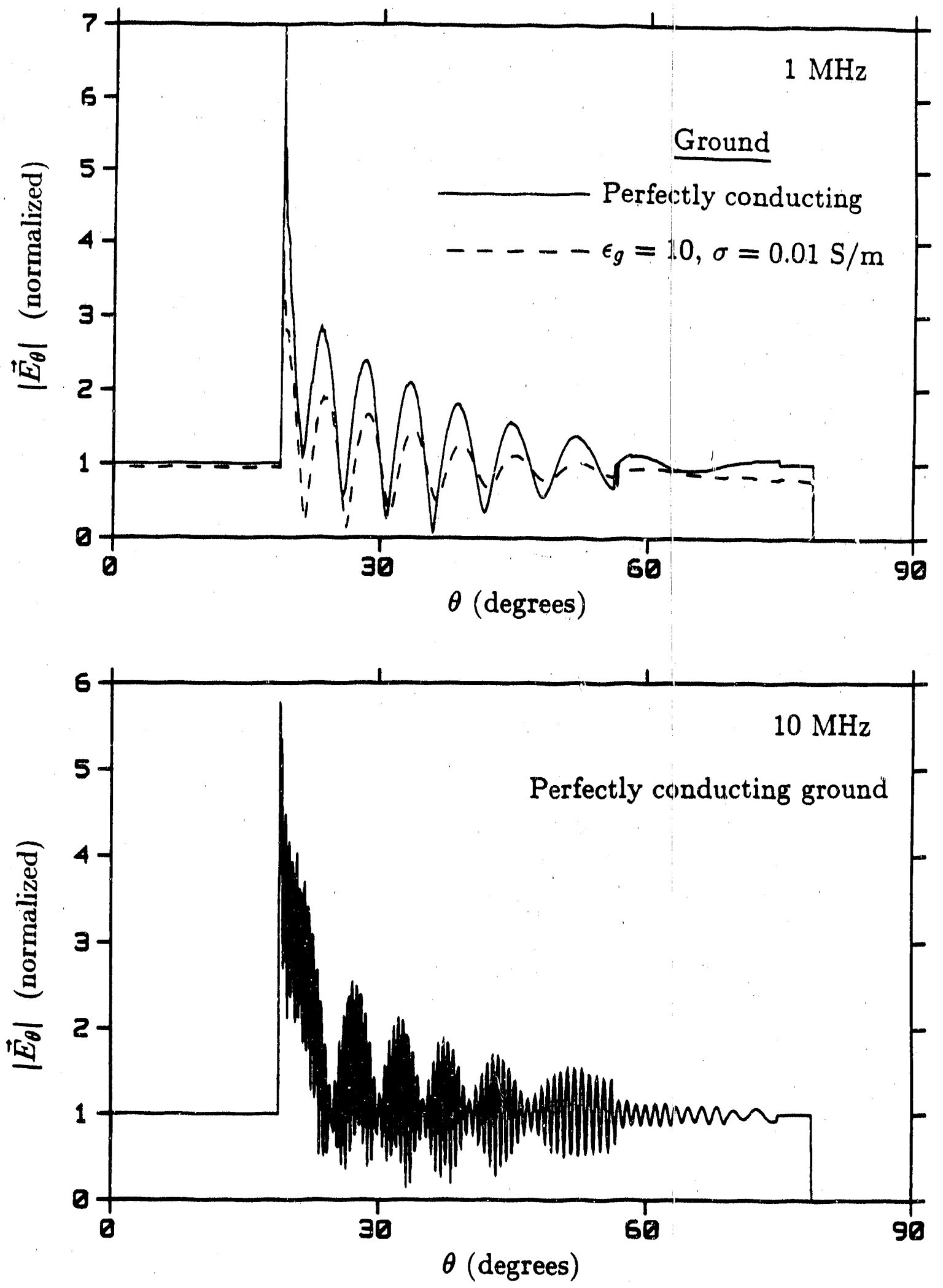

Fig. 7. Radiation pattern for normalized $E_{0}$ over the perfectly conducting Gaussian ridge in Fig. 5 . 




Fig. 8. Comparison of normalized radiation patterns for a Gaussian ridge and a round Gaussian hill.

Another effect that can be examired with the GO model is that of curvature of the terrain surface normal to the plane of propagation. The two dimensional Gaussian ridge was modeled in the GO code as a long strip, one cell wide and many cells long, to take advantage of the two dimensional geometry. This model is easily modified to introduce curvature in the transverse direction, while the code still scans an essentially two dimensional model. The effect of transverse curvature is to increase the divergence of the reflected ray and hence reduce the strength of the scattered field. In Fig. 8 the field scattered by the two dimensional Gaussian ridge is compared with that scattered by a round Gaussian hill having the same cross section. The added ray divergence is seen to reduce the scattered field by a factor of about two for high angles of incidence. The difference disappears toward grazing incidence. This result indicates that a correction may be needed for models that can only treat two-dimensional terrain.

Radiation patterns of $E_{\theta}$ for a short vertical dipole on the surface of the ground, $500 \mathrm{~m}$ from a round Gaussian hill of height $150 \mathrm{~m}$ and width $w=500 \mathrm{~m}$ are shown in Fig. 9. Similar patterns for $E_{\phi}$ due to a horizontal dipole are shown in F:g. 10. The ground parameters were $\epsilon_{g}=10, \sigma=0.01 \mathrm{~S} / \mathrm{m}$ and the dipoles had a current moment of 1 Amp-m. Although the reflection coefficient is generally larger for horizontal polarization, the scattering from the hill, at low frequencies, is larger for vertical polarization. This is because the out of phase image of the horizontal source results in a smaller incident field on the hill than for the vertical source. With the source on the ground, the source and its image in the ground coincide. The direct and image contributions were traced as a single ray to the hill. As $\theta$ increases beyond about 58 degrees the middle ray, as shown in Fig. 2, 
becomes blocked, and only the ray from the source and that reflected near the top of the hill reach the receiver. The latter is weakened by a large divergence near grazing incidence. Hence just before total shadowing the field is that for the source on a flat ground plane.

The stair-step effect near grazing incidence on the hill is a result of the calculation of the divergence factor for the triangular ray tubes. As the specular point moves toward the top of the hill it crosses triangles with the base alternately toward or away from the source. As the ray tube divergence transverse to the plane of propagation decreases toward $t$ \%. point of grazing incidence the triangles with the base away from the source have smalir $r$ areas than those with base toward the source. This effect would be less noticeable if the ray was not incident normal to the edges of the triangles.

Radiation patterns are shown in Figures 11 and 12 for the vertical and horizontal dipole sources of Figures 9 and 10 elevated $100 \mathrm{~m}$ above the ground. The effect for either polarization is to greatly increase the field scattered from the hill. With this source elevation the ray reflecting closest to the source does not undergo a second reflection from the hill. Also, the middle ray shown in Fig. 2 does not berome blocked by the hill top as it did with the source on the ground. Instead it merges with the lower ray in a second caustic around $\theta=53$ degrees. Beyond this angle only the direct ray from the source and the ray reflected near the top of the hill reach the source. Since the latter is weakened by divergence, the field just before total shadowing is that of the source in free space.

The patterns for the source and hill configurations of Figures 9 through 12 are plotted in Figures 13 through 16 normalized to the field of the same source over flat ground.

\section{Conclusion}

The problem of modeling the radiation from $\mathrm{HF}$ antennas sited in irregular terrain has been investigated. This study was part of an ongoing project to characterize terrain effects at $\mathrm{HF}$. The primary interest is in skywave communication involving antennas near hills, valley's and cliffs. Methods considered for modeling include geometrical optics, Geometrical Theory of Diffraction, surface integration (physical optics), Volterra integral equations for field propagation, integral equations for surface currents and finite-difference time domain solutions.

A computer code was developed to apply GO for modeling arbitrary terrain surfaces. The method of ray launching, developed hy Mittra and Rushdi [1], was extended to treat concave and convex surfaces with finite conductivity. This code is capable of modeling three dimensional terrain and is expected to provide useable predictions for field in illuminated regions. The lack of surface wave and diffraction limits the accuracy of GO for radiation angles grazing the ground. The code yields zero field in shadow regions. At present, only singly reflected rays are traced, although for a source on the ground surface, outside of the arbitrary terrain grid, the ray from the source and its image can be traced as a single ray. Extension of this code to trace at least doubly reflected rays should be considered in the future. 
A source cannot be located on the arbitrary terrain surface in the GO model, since the interpolation formulas would fail. For the results shown here with a source on the surface, the source was located over flat ground, outside of the region of irregular terrain. The arbitrary terrain model can be used with a source on the surface and a distant receiver by invoking reciprocity and tracing the ray from the receiver to the source at the surface. This would require some simple changes in the present code. If the point on the surface is on a curve, such as the side or top of a hill, the radius of curvature must be much larger than the wavelength for GO to be valid. The model would still have limited usefulness in this case, since there would be total shadowing beyond 90 degrees from the surfare normal.

The GO code was tested for an antenna near a Gaussian hill or ridge. Results were generally in agreement with those of Berry [5] obtained by integrating the surface fields from the solution of a Volterra integral equation. However, the GO result for the interference pattern produced by the ridge ends abruptly at a caustic surface, while Berry shows the pattern extending somewhat in front of the ridge. The integral equation solution gives a valid result in the shadow of the ridge, where the GO field is zero. A significant difference was seen between the scattering by a ridge with the conductivity of moderately good earth and a perfectly conducting surface. Also, the transverse component of curvature on a hill, as compared to a two dimensional ridge, was shown to be important at high angles of incidence.

There are a number of further developments that should be considered for the GO code to make it a more generally useful modeling tool. The need for multiple reflections and the capability of locating a source on the terrain surface were mentioned above. Also, the reflection coefficients could be modified to take account of surface roughness, using results from [42]. Diffracted fields at either single or multiple edges would be a valuable addition to predict fields in regions where the source is shadowed by the terrain. Diffraction would greatly increase the complexity of the code, however.

Practical applications of this code will require actual HF antennas as a source, rather than the infinitesimal dipole that was used here. The source field is supplied by a single subroutine that could easily be replaced by a routine to compute radiated field of a current distribution supplied by an antenna modeling code such as NEC. A procedure to transform currents computed on a flat ground plane to an inclined surface of irregular terrain is given as an appendix to this report. Also, a convenient means of entering the terrain profile is needed. In the present code the elevations and normal vectors describing the terrain are generated in a subroutine from a mathematical function. A number of these terrain subroutines can be included in the code and selected by an input parameter. For practical use, a means is needed for entering real terrain data, possibly using a CAD program.

Due to the complexity of the terrain modeling problem and the variety of conditions that may occur, neither the GO code nor other techniques discussed appear capable of treating all cases of interest. Other models, based on the Volterra integral equation, GTD and the finite-difference time domain solution should be developed and validated for a variety of antenna siting problems. In addition to determining appropriate modeling lechniques, the acceptable levels of error must be established, taking into account such 
factors as magnitude of the terrain effect, the uncertainty in terrain profile measurements and the requirements for modeling the communication link.

An important step in developing and validating such models is comparison with measured data. Little experimental data is now available on radiation from antennas in irregular terrain. A measurement program is being undertaken on another part of this project for the U. S. Navy. The results of the GO code and other available models will be compared with this data when it is available. More measured data appears to be available for ground wave propagation than for skywave. Bach Andersen [43] measured ground wave and low-angle skywave for varying distances from a coastline at 14 and $25 \mathrm{MHz}$. Ot.t, et al. $[27,44]$ published a comparison of measurements with results from the Volterra integral equation solution for ground wave propagation at 60 and $0.74 \mathrm{MHz}$ over a mixed land and sea path. Knight and Thoday [35] measured low angle skywave at $0.845 \mathrm{MHz}$ near a coastline. Also, Barker. Taylor and Hagn [45] measured the radiation patterns of several field-expedient $\mathrm{HF}$ antennas over various types of terrain, including hills.

Validation of models for terrain effects is probably at least as difficult as the model development, due to the difficulties and uncertainties in characterizing the terrain and making measurements. A lot more work will be needed to develop an adequate set of modeling tools and to validate them and gain experience in terrain modeling and measurement. 

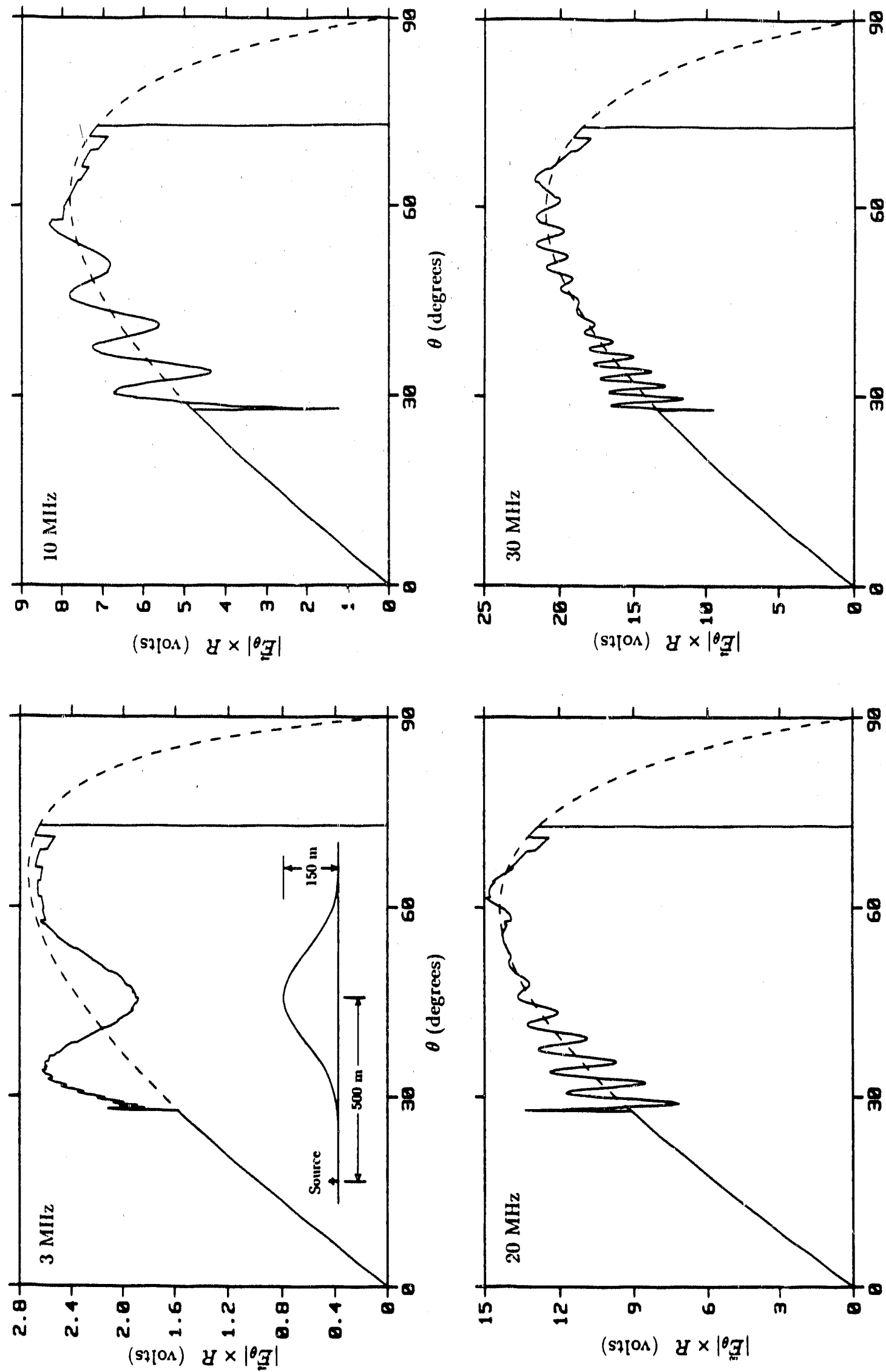

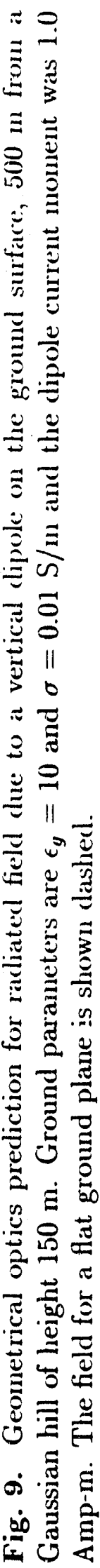



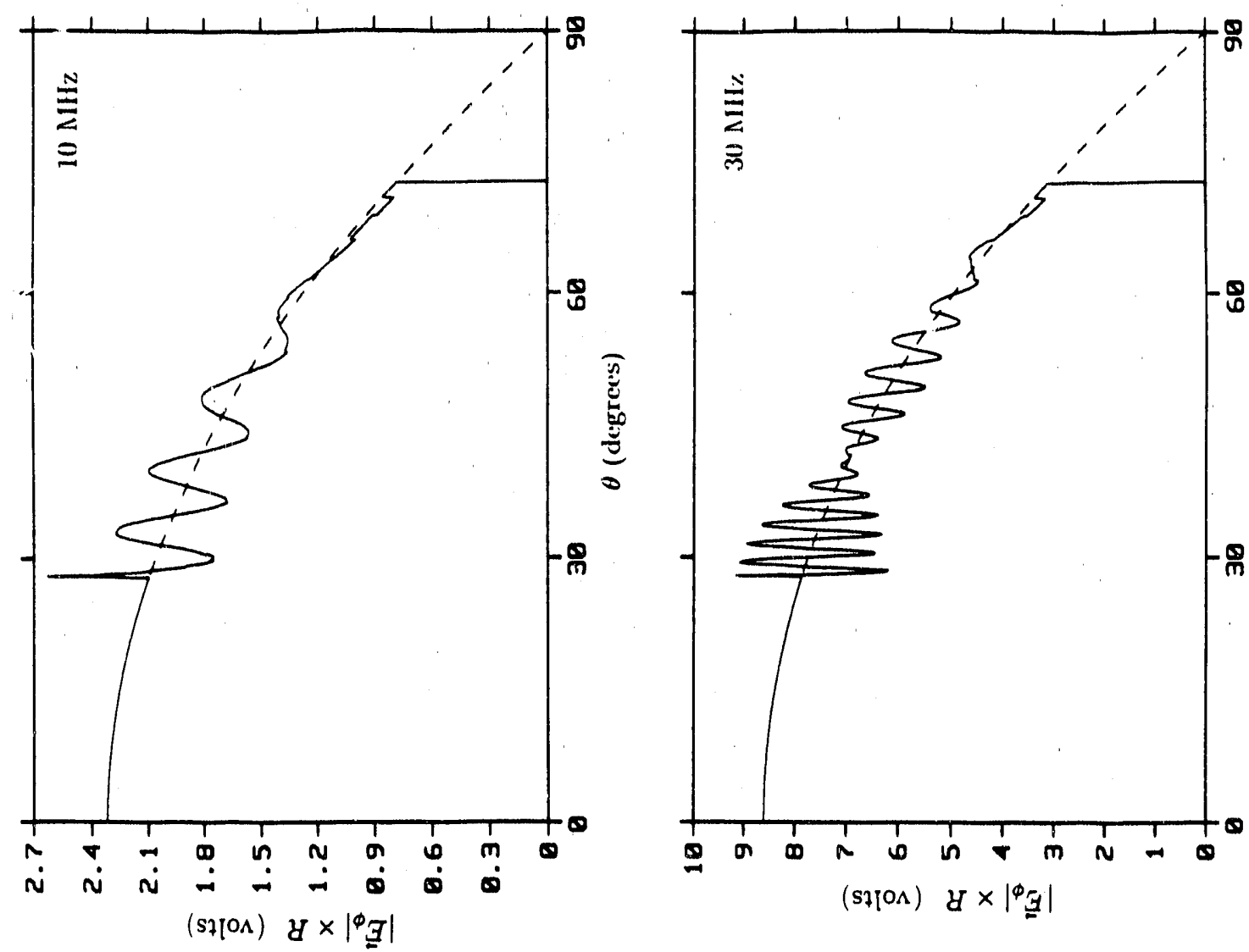

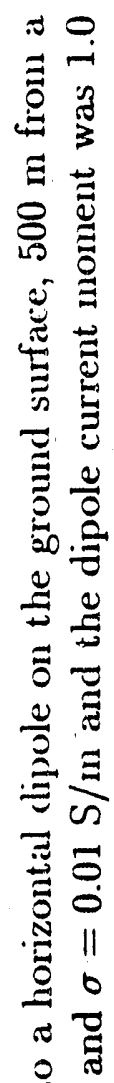
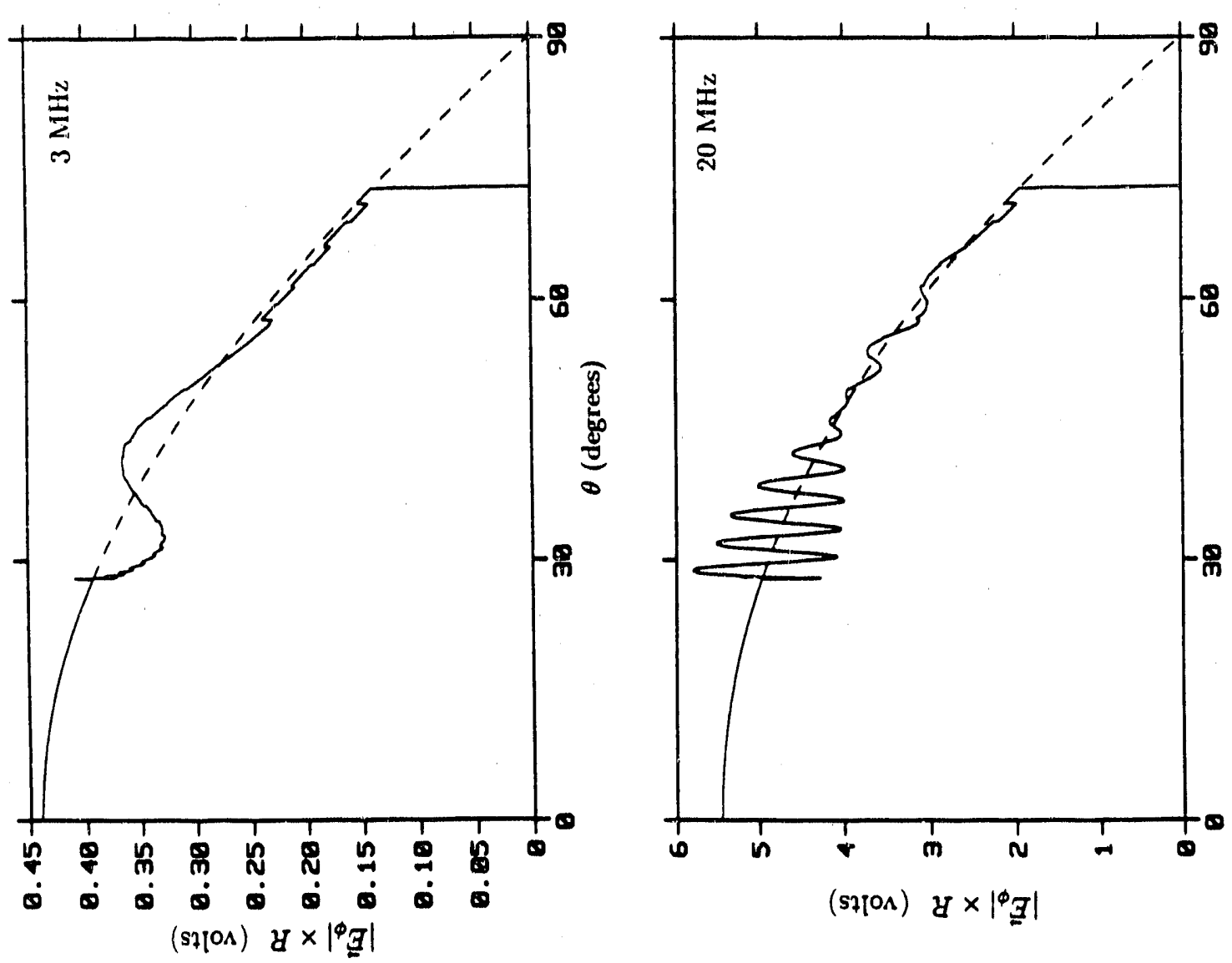

으

$\stackrel{\varrho}{\Xi}$

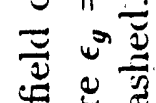

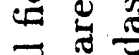

उ.

胥 导

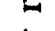

놈

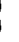

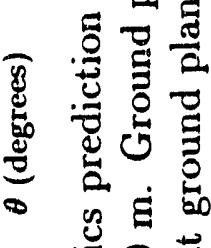

.

넌

తี

过

늉

ญ

ㅇำ

(s7[0s) $y \times\left.\right|^{\phi} g \mid$

承菌 

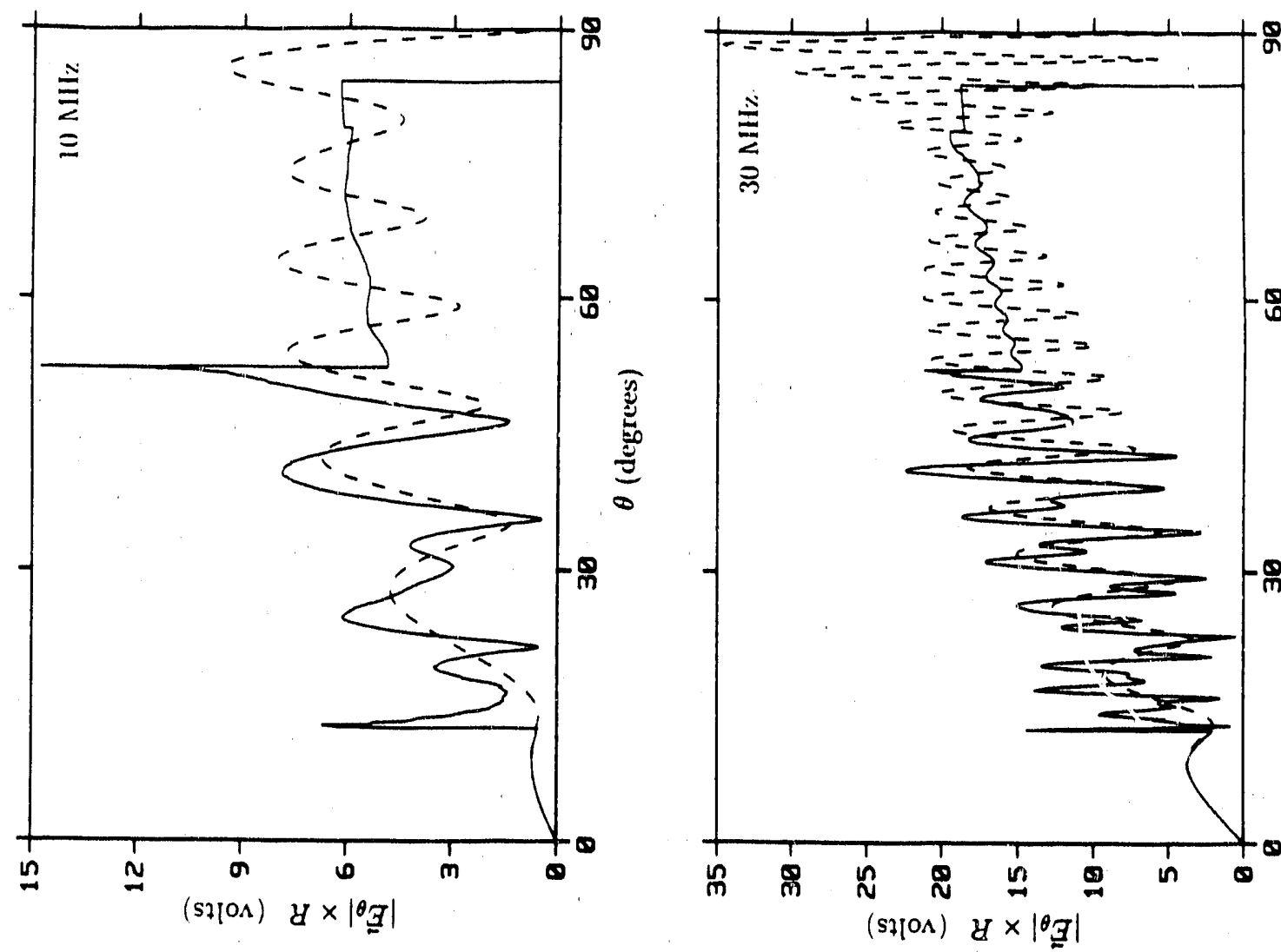

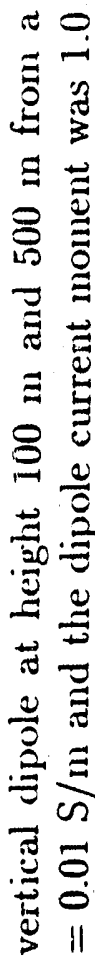

* 0

๑ 을

$\stackrel{\Xi}{\Xi}$
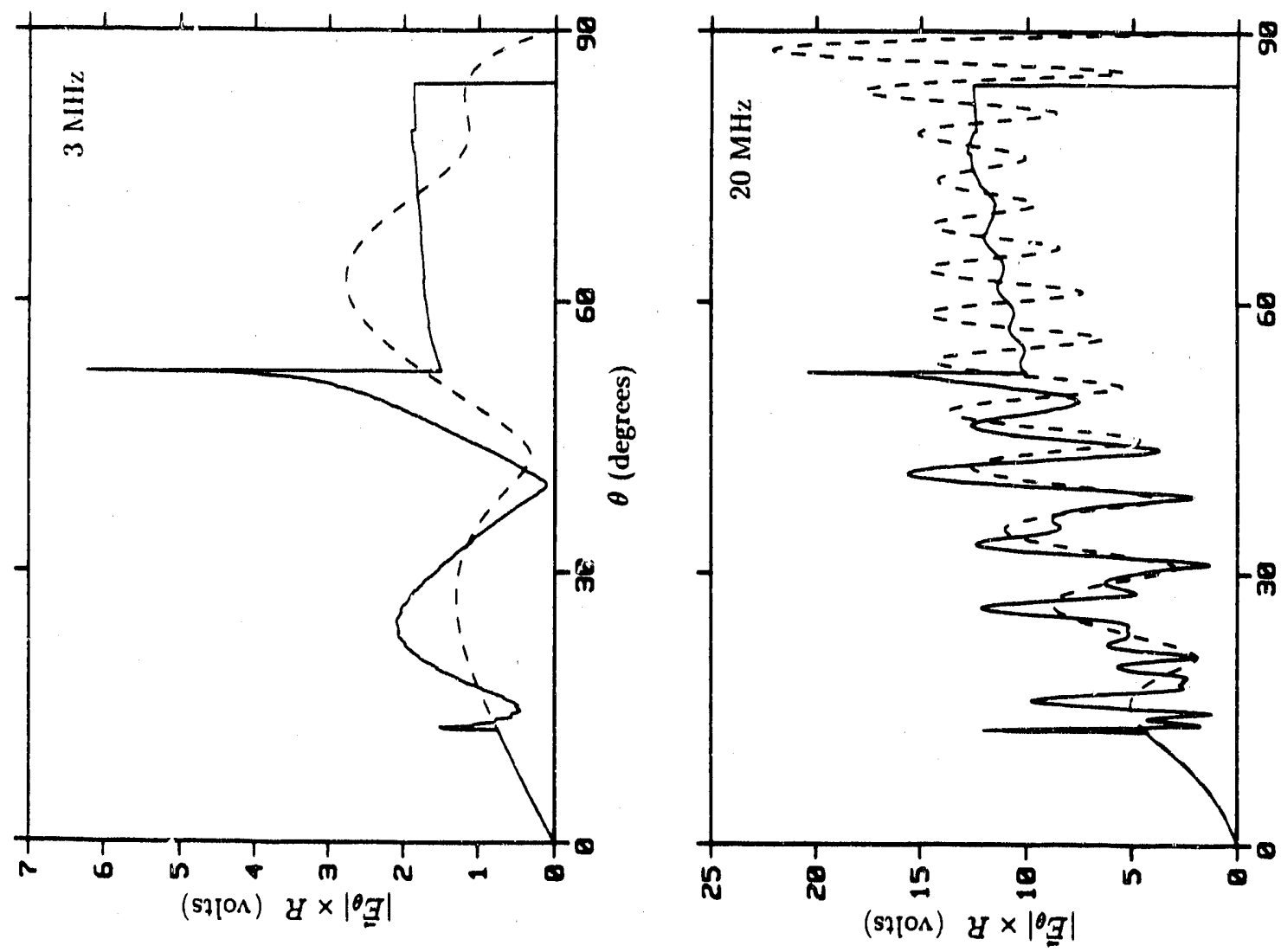

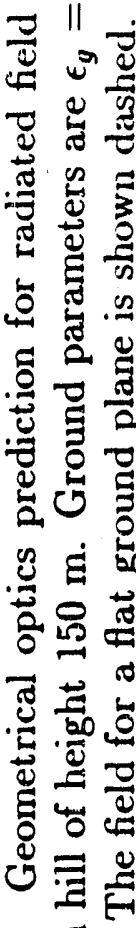

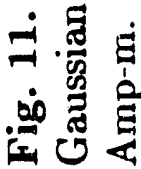



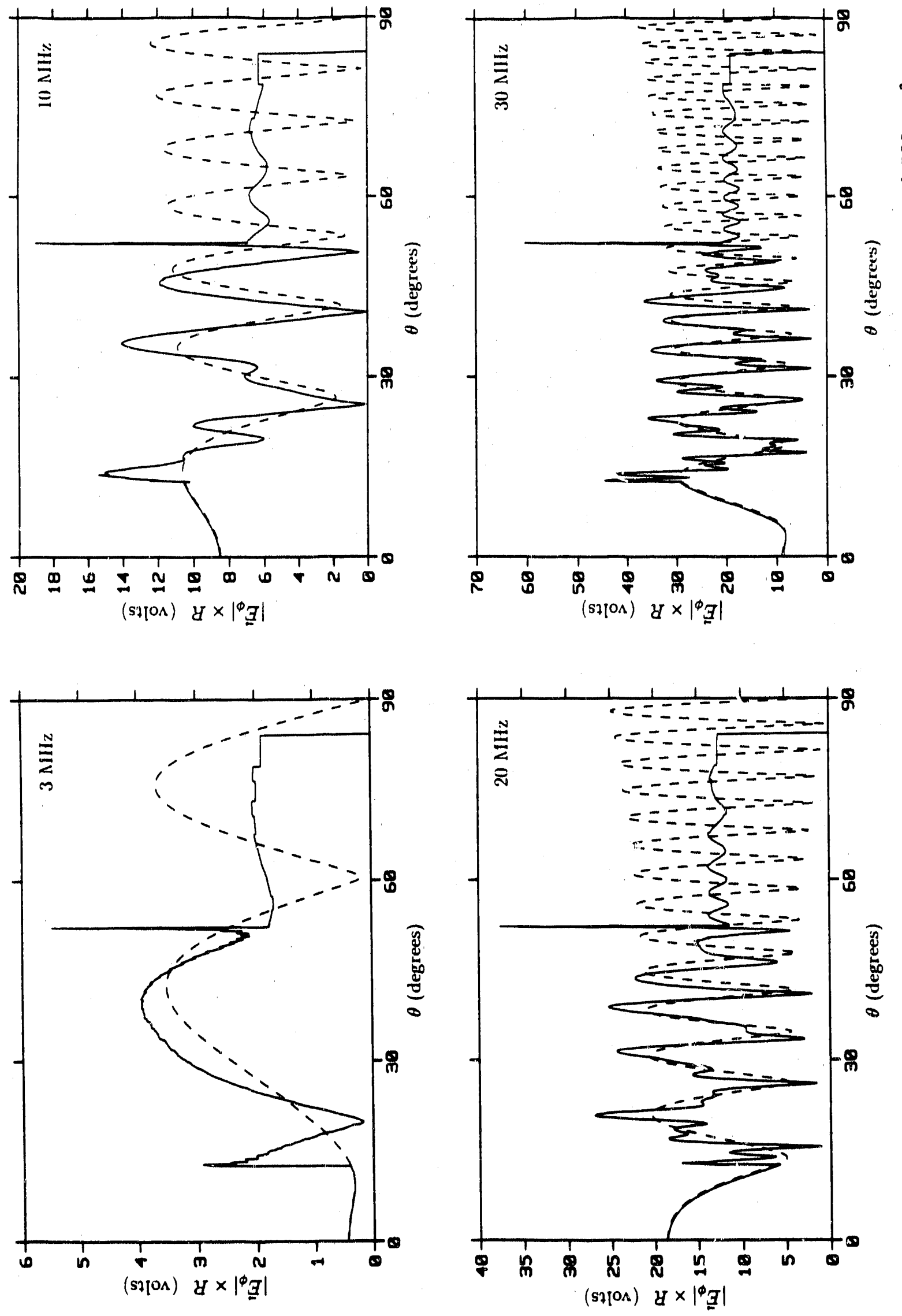

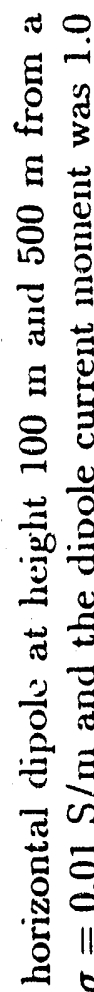

을

$\vartheta$

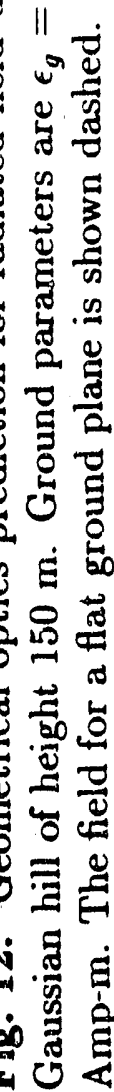




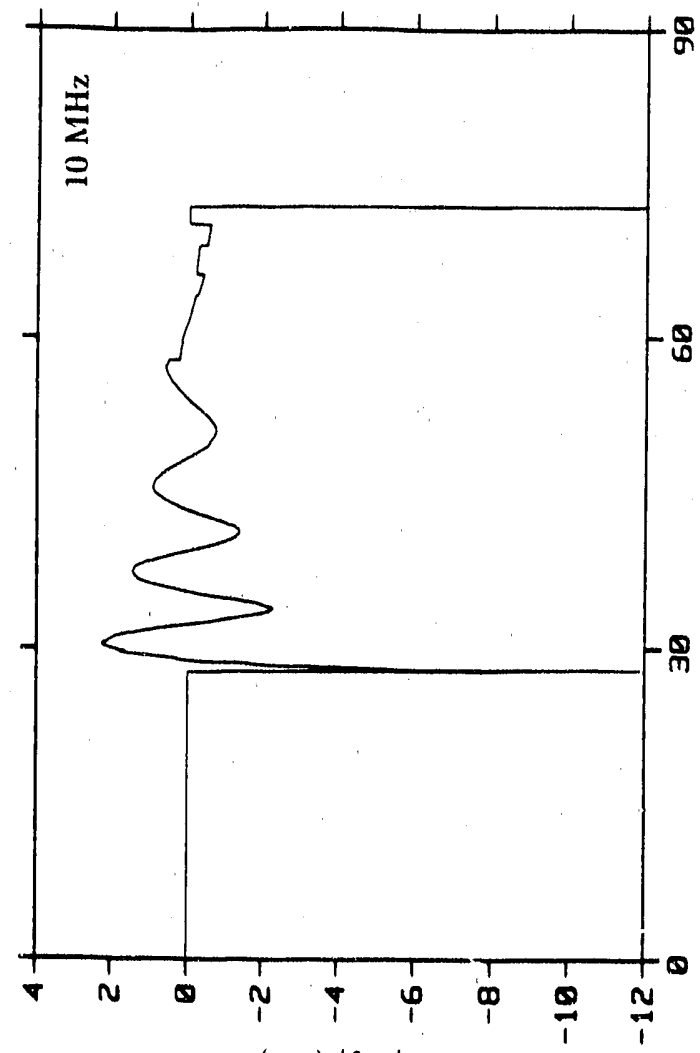

(ap) $\left|\boldsymbol{\theta}_{\mathcal{M}}\right|$

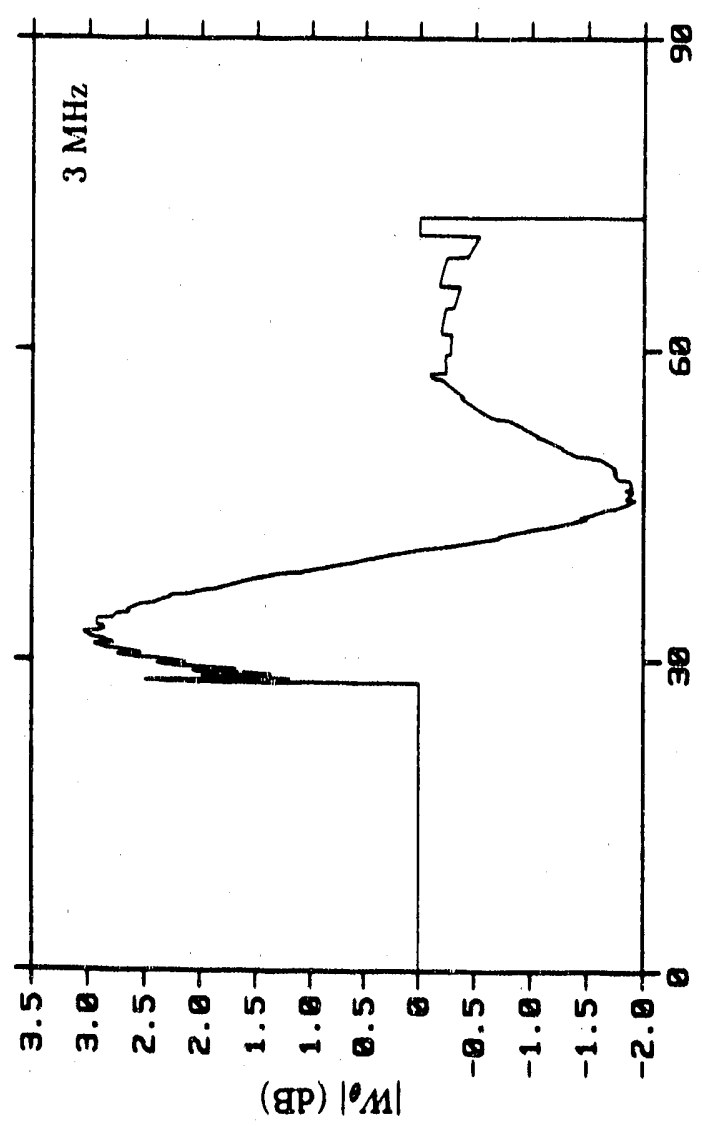

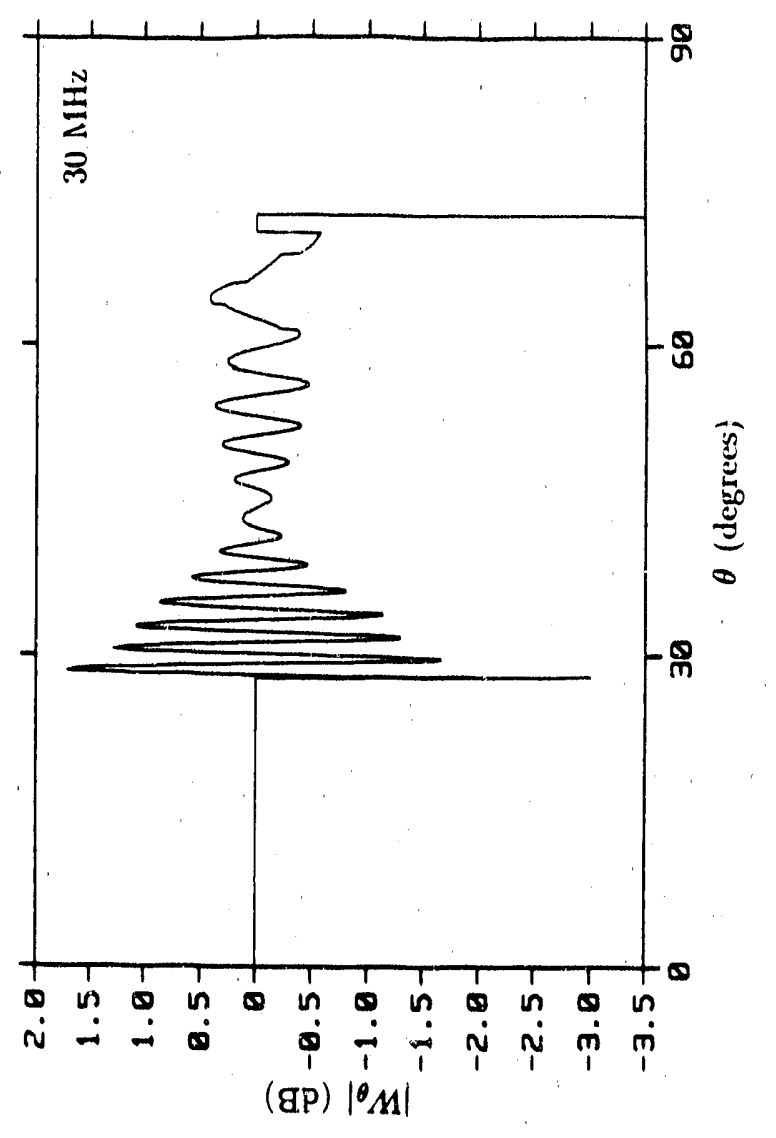

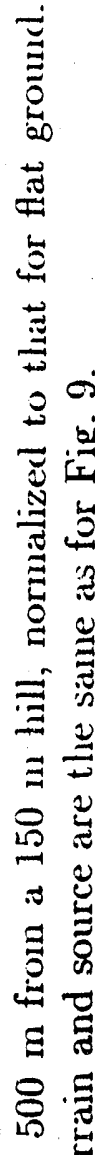

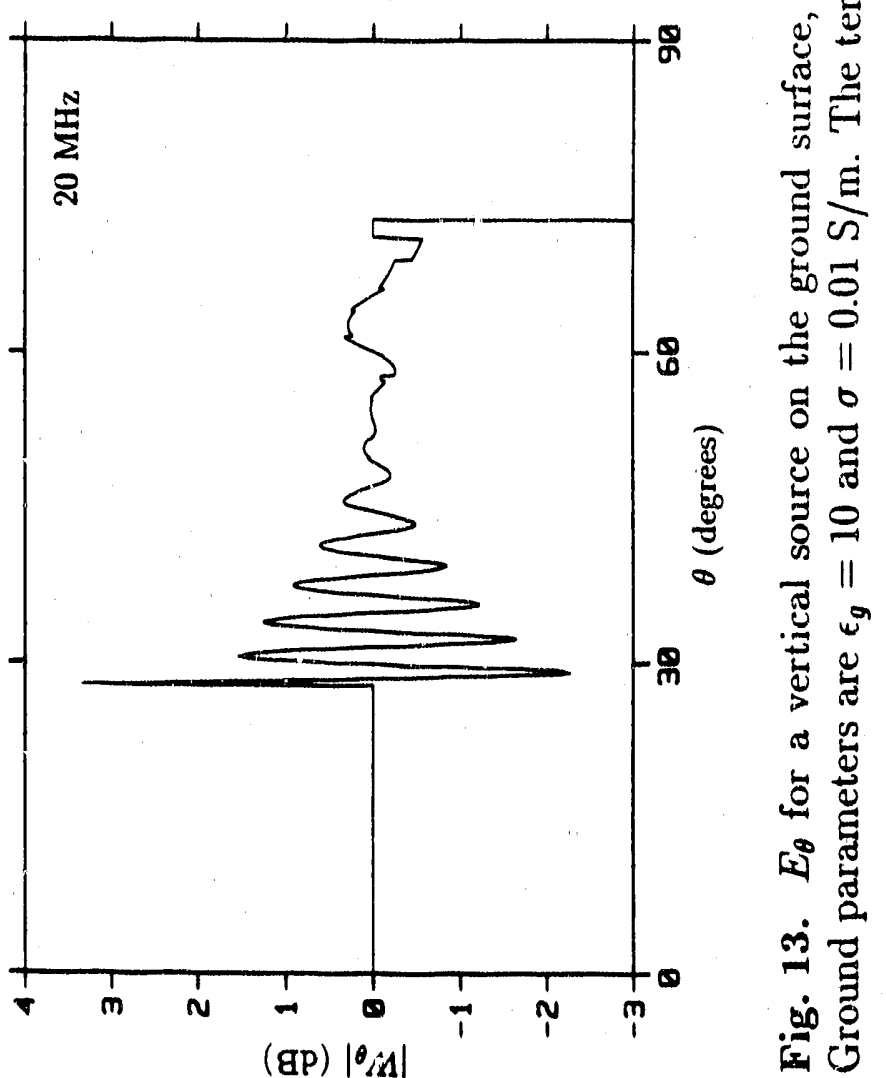




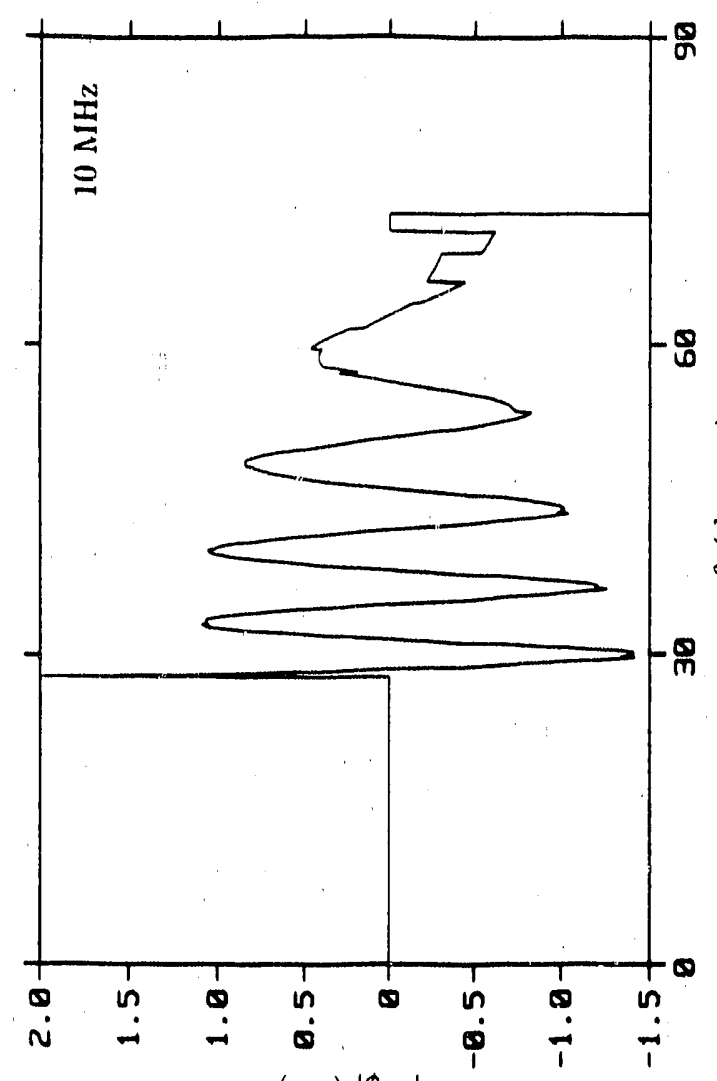

(gp) $\left|{ }^{\Phi} M\right|$

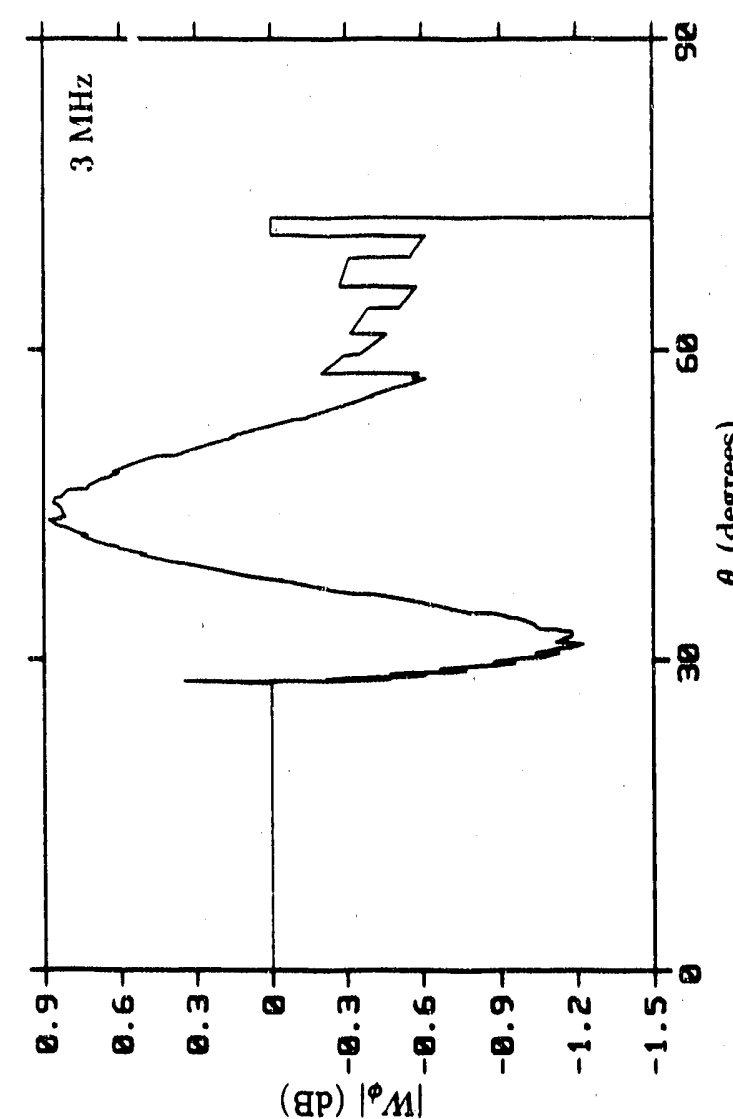

(gp) $|\Phi M|$
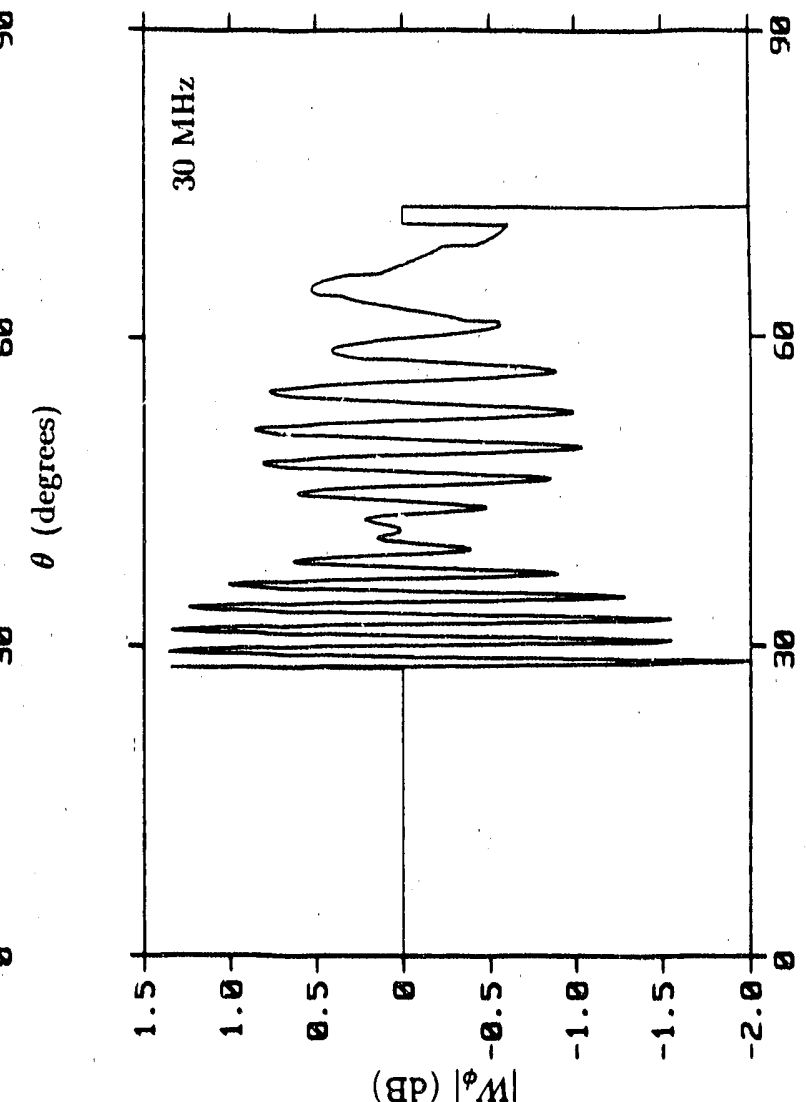

(gP) $\left|{ }^{\phi} M\right|$

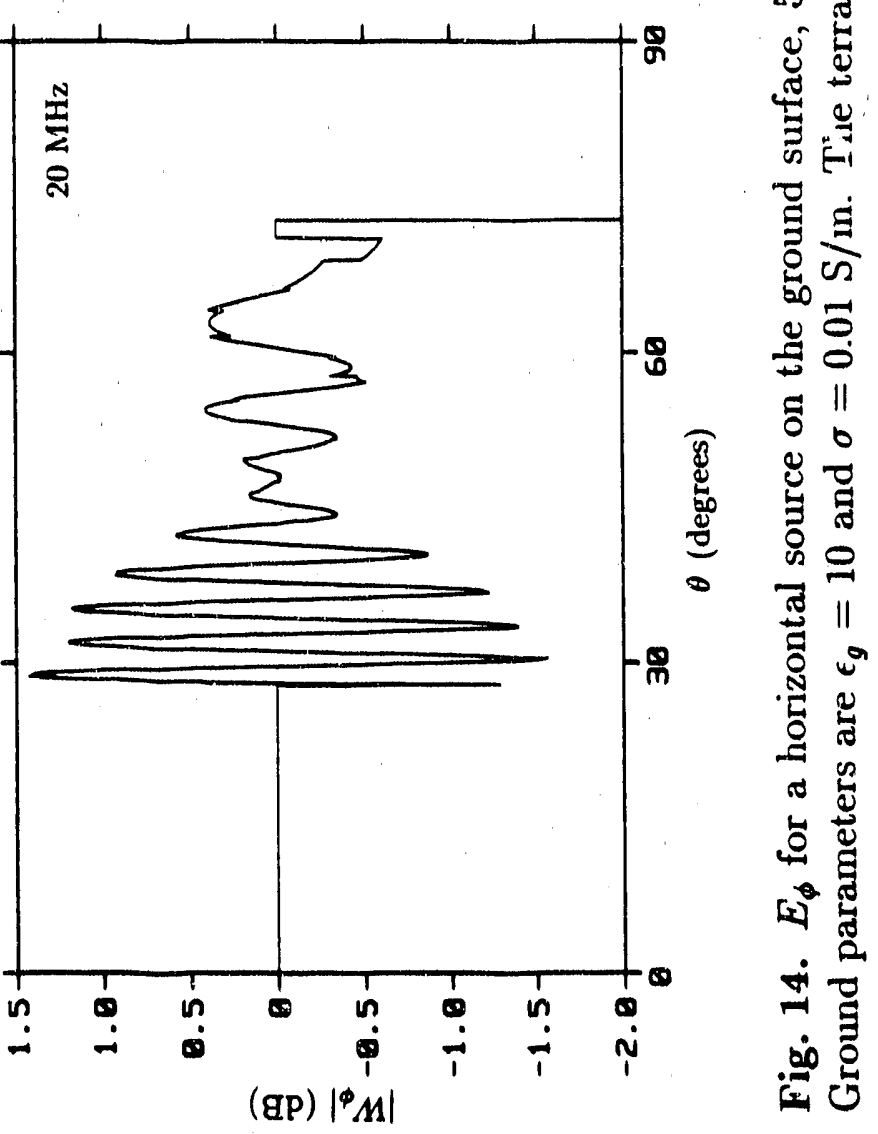




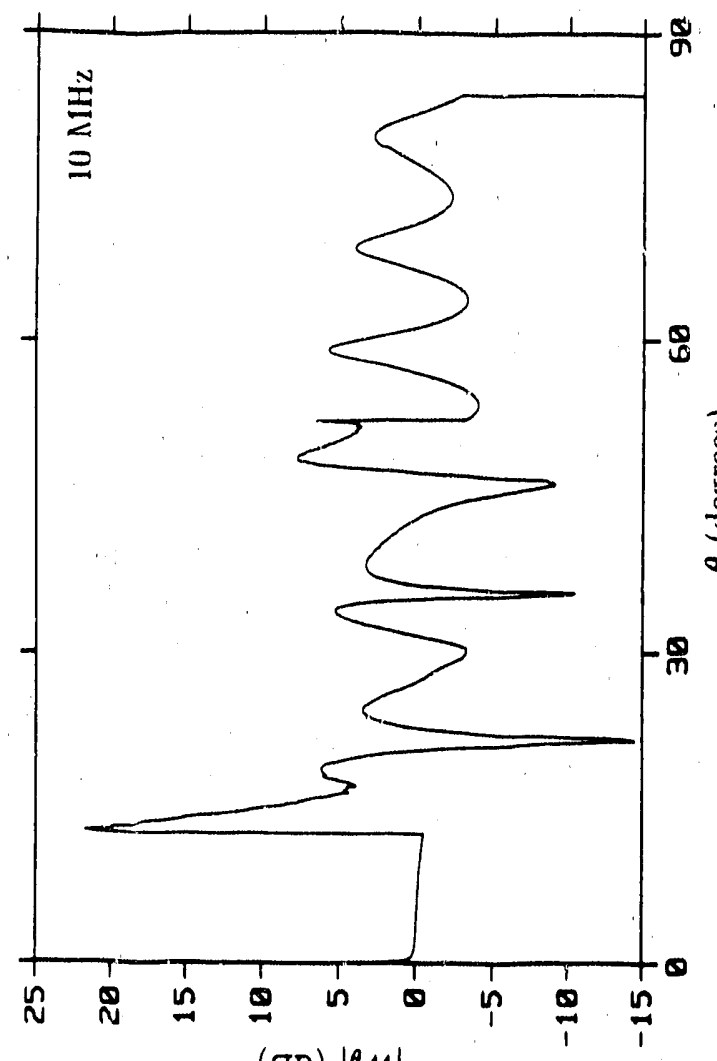

(gP) $|\theta M|$

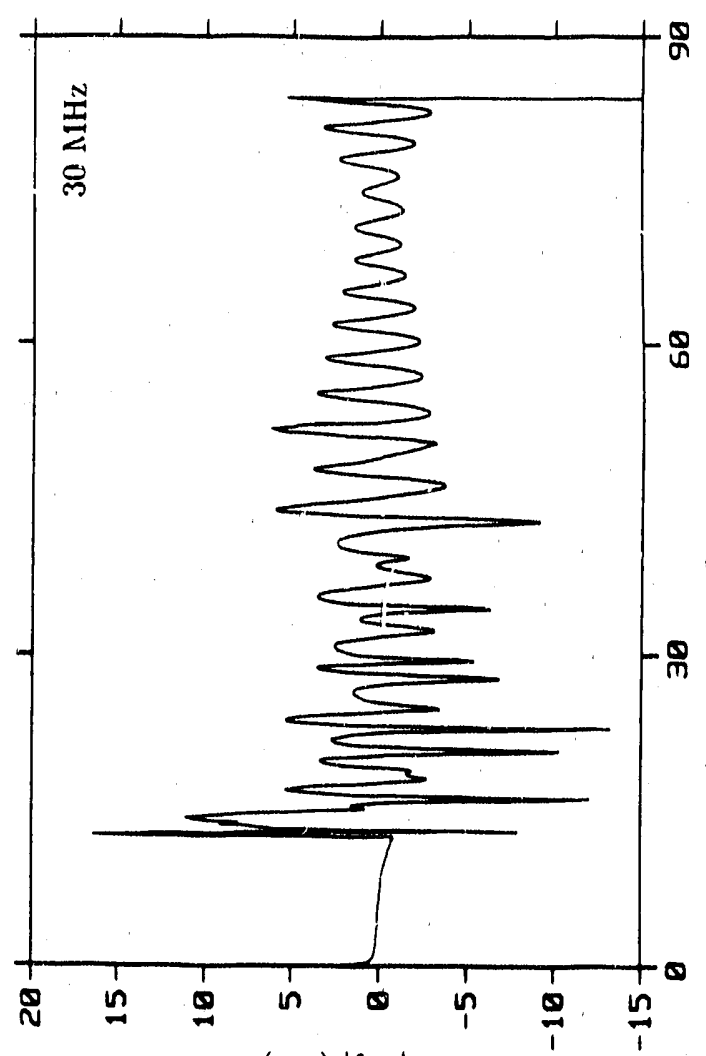

(gp) $\left|\theta^{\theta} M\right|$
క్

苛

$\stackrel{1}{1}$

D

즌

尔

今

三苛

E

윤

त

苞总

园

8 종

ชั

สำ

\&

马욜

욜

0 $\frac{\pi}{0}$

क

\&

巳一

0

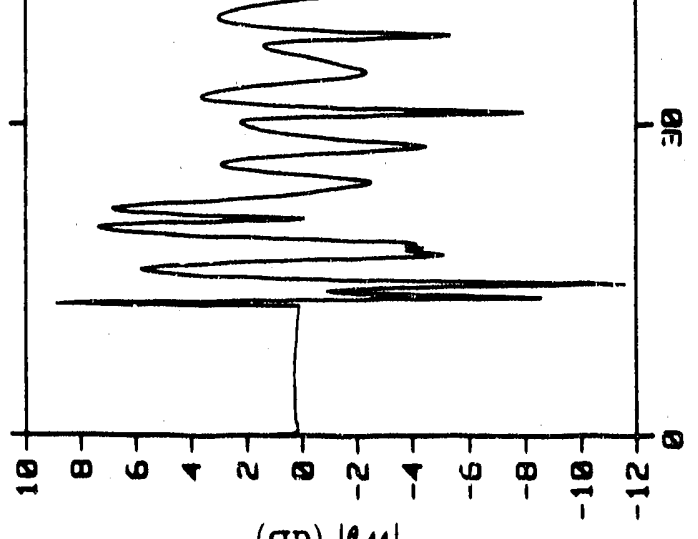

(ap) $\left|\theta^{M}\right|$ 

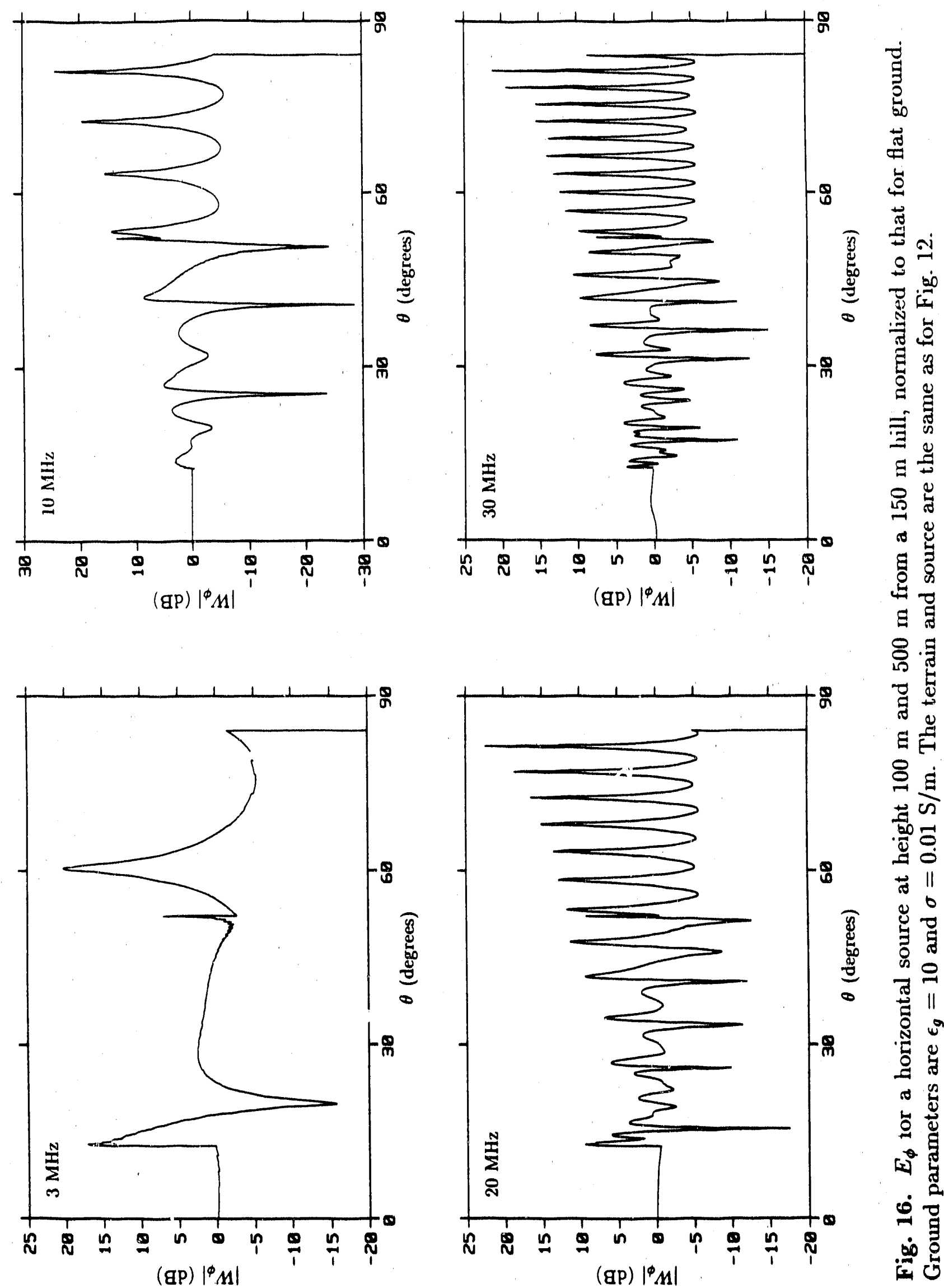


\section{References}

[1] R. Mittra and A. Rushdi, "An Efficient Approach for Computing the Geometrical Optics Field Reflected from a Numerically Specified Surface," IEEE Trans. Antennas and Propagation, Vol. AP-27, No. 6, pp. 871-877, Nov. 1979.

[2] G. J. Burke and A. J. Poggio, Numerical Electromagnetics Code (NEC) - Method of Moments, Lawrence Livermore National Laboratory, Rept. UCID-18834, Jan. 1981.

[3] G. J. Burke and E. K. Miller, "Modeling Antennas Near to and Penetrating a Lossy Interface," IEEE Trans. Antennas and Propagation, Vol. AP-32, pp. 1040-1049, Oct. 1984.

[4] J. C. Logan and J. W. Rockway, The New MININEC (Version \&): A Mini-Numerical Electromagnetic Code, Naval Ocean Systems Center TD 938, September 1986.

(5] L. A. Lherry, "Radio Propagation over a Gaussian-Shaped Ridge," IEEE Trans. An. tennas «nd Propagation, Vol. AP-15, pp. 701-703, Sept. 1967.

[6] R. H. Ott and L. A. Berry, "An Alternative Integral Equation for Propagation over Irregular Terrain," Radio Science, Vol. 5, No. 5, pp. 767-771, May 1970.

[7] R. H. Ott, "An Alternative Integral Equation for Propagation over Irregular Terrain, 2," Radio Science, Vol. 6, No. 4, pp. 429-435, April 1971.

[8] G. A. Deschamps, "Ray Techniques in Electromagnetics," Proc. of the IEEE, Vol. 60, No. 9, pp. 1022-1035, Sept. 1972.

[9] C. A. Balanis, Advanced Engineering Electromagnetics, John Wiley and Sons, New York, NY, 1989.

[10] Y. T. Lo and S. W. Lee, Antenna Handbook, Van Nostrand Reinhold Co., New York, NY, 1988.

[11] D. E. Kerr, Propagation of Short Radio Waves, MIT Radiation Laboratory Series, McGraw-Hill Co., New York, NY, 1951.

[12] J. B. Keller, "Geometrical Theory of Diffraction," Journal of the Optical Soc. of Amer., Vol. 52, No. 2, pp. 116-130, Feb. 1962.

[13] R. G. Kouyoumjian, "Asymptotic High-Frequency Methods," Proc. IEEE, Vol. 53, pp. 864-876, Aug. 1965.

[14] R. G. Kouyoumjian and P. H. Pathak, "A Uniform Geometrical Theory of Diffraction for an Edge in a Perfectly Conducting Surface," Proc. IEEE, Vol. 62, No. 11, pp. 1448-1461, Nov. 1974.

[15] J. C. Schelleng, C. R. Burrows and E. B. Ferrell, "Ultra-Short-Wave Propagation," 
Proc. of the IRE, Vol. 21, No. 3, pp. 427-463, March 1933.

[16] R. J. Luebbers, "Finite Conductivity Uniform GTD Versus Knife Edge Diffraction in Prediction of Propagation Path Loss," IEEE Trans. Antennas and Propagation, Vol. AP-32, pp. 70-76, Jan. 1984.

[17] R. J. Luebbers, "Propagation Prediction for Hilly Terrain U'sing GTD Wedge Diffraction," IEEE Trans. Antennas and Propagation, Vol. AP-32, pp. 9ó1-955, Sept. 1984.

[18] J. R. Wait, "Diffraction and Scattering of the Electromagnetic Groundwave by Terrain Features," Radio Science, Vol. 3, No. 10, pp. 995-1003, Oct. 1968.

[19] J. R. Wait and K. P. Spies, "Radio Propagation Over a Dylindrical Hill Including the Effect of a Surmounted Obstacle," IEEE Trans. Anteninas and Propagation, Vol. AP-16, No. 6, pp. 700-705, Nov. 1968.

[20] R. J. Marhefka and W. D. Burnside, "Numerical Electromagnetics Code - Basic Scattering Code (Version 2), Part I: User's Manual," The Ohio State University, ElectroScience Lab. Tech. Report 712242-14, Dec. 1982.

[21] R. J. Marhefka, "Numerical Electromagnetics Code - Basic Scattering Code (Version 2), Part II: Code Manual," The Ohio State University, ElectroScience Lab. Tech. Report 712242-15, Dec. 1982.

[22] R. J. Marhefka and J. W. Silvestro, "Near Zone - Basic Scattering Code; User's Manual with Space Station Applications," The Ohio State University, ElectroScience Lab. Tech. Report 716199-13, March 1989.

[23] G. D. Monteath, "Application of the Compensation Theorem to Certain Radiation and Propagation Problems," Proc. IEE, Vol. 98, Pt. IV, 1951.

[24] V. V. Liepa, "Numerical Approach for Predicting Radiation Patterns of HF-VHF Antennas over Irregular Terrain," IEEE Trans. Antennas and Propagation, Vol. AP-16, pp. 273-274, March 1968.

[25] H. Page and G. D. Monteath, "The Vertical Radiation Patterns of Medium-Wave Broadcasting Aerials," Proc. IEE, Vol. 102B, pp. 279-297, M.ay 1955.

[26j G. A. Hufford, "An Integral Equation Approach to the Problem of Wave Propagation over an Irregular Tezrain," Quart. Appl. Math., Vol. 9, No. 4;, pp. 391-404, 1952.

[27] R. H. Ott, L. E. Vogler and G. A. Hufford, "Ground-Wave Propagation over Irregular Inhomogeneous Terrain: Comparisons of Calcilations and Measurements," IEEE Trans. Antennas and Propagation, Vol. AP-27, No. 2, pp. 284-286, March 1979.

[28] D. A. Hill, "HF Ground Wave Propagation over Forested and Built-up Terrain," U. S. Dept. of Commerce, NTIA Report 82-114, 1982. 
[29] Z. Wu, T. S. Maclean, D. J. Bagwell and M. J. Mehler, "Propagation over an Inhomogeneous Irregular Surface," Radio Science, Vol. 23, No. 1, pp. 33-40, Jan.-Feb. 1988.

[30] R. M. Bevensee, "Compensation-Theorem Analyses of Ground Wave Propagation over Irregular, Inhomogeneous Terrains," Journal of Atmospheric and Terrestrial Physics, Vol. 51, No. 9/10, pp. 797-810, 1989.

[31] R. H. Ott, "The Radiation Fields from HF Costal Antennas," U. S. Dept. of Commerce, OT Tech. Memorandum 74-174, 1974.

[32] G. D. Monteath, Application of Electromagnetic Reciprocity Principle, Pergamon, New York, 1973.

[33] J. Arnbak and J. Bach Andersen, "Skywave Propagation over a Multisection Plane Ground," Electronics Letters, Vol. 7, No. 23, pp. 685-686, 18 Nov. 1971.

[34] J. C. Arnbak, "Ground-loss Profile along a Multi-Section Path of a Sky Wave," Paper 26 for AGARD Spring 1974 Meeting, AGARD-CPP-144, The Hague, Netherlands, March 25-29, 1974.

[35] P. Knight and R. D. C. Thoday, "Influence of the Ground Near Transmitting and Receiving Aerials on the Strength of Medium-Frequency Sky Waves.," Proc. IEE, Vol. 116, No. 6, June 1969.

[36] A. J. Poggio and E. K. Miller, "Integral Equation Solutions of Three-Dimensional Scattering Problems," Chapt. IV in Computer Techniques for Electromagnetics, edited by R. Mittra, Pergamon Press, NY, 1973.

[37] S. M. Rao, D. R. Wilton and A. W. Glisson, "Electromagnetic Scattering by Surfaces of Arbitrary Shape," IEEE Trans. on Antennas and Propagation, Vol. AP-30, No. 3, May, 1982.

[38] W. A. Johrson, D. R. Wilton and R. M. Sharpe, "Modeling Scattering from and Radiation by Arbitrary Shaped Objects with the Electric Field Integral Equation Triangular Surface Patch Code," Electromagnetics, Vol. 10, pp. 41-63, 1990.

[39] R. Corry and G. Lane, "Numerical Modeling Technique for Antenna Siting in Mountainous Terrain," Headquarters, USA CEEIA, Ft. Huachuca AZ, Tech. Report EMEOPED-81-6, May 1981.

[40] K. S. Yee, "Numerical Solution of Initial Boundary Value Problems Involving Maxwell's Equation in Isotropic Media," IEEE Trans. Antennas and Propagation, Vol. AP-14, pp. 302-307, May 1966.

[41] R. R. McLeod, "Temporal Scattering And Response Software - Users' Manual Version 2.1 (R)," Lawrence Livermore National Laboratory, UCRL-MA-104861, Sept. 1990. 
[42] P. Beckmann and A. Spizzichino, The Scattering of Electromagnetic Waves from Rough Surfaces, Pergamon Press, 1963.

[43] J. Bach Andersen, "Reception of Skywave Signals Near a Coastline," Journal of Re. search of the National Bureau of Standards - D. Radio Propagation, Vol. 67D, No. 3, pp. 325-330, May-June 1963.

[44] R. H. Ott, "Ground Wave Propagation over Irregular, Inhomogeneous Terrain: Comparisons of Calculations and Measurements at Frequencies from $121 \mathrm{kHz}$ to $50 \mathrm{MHz}$," Paper No. 8 in AGARD Conference Proceedings: Terrain Profiles and Contours in E. M. Propagation, AGARD-CPP-269, Sept. 10-14, 1979.

[45] G. E. Barker, J. Taylor and G. H. Hagn, "Summary of Measurements and Modeling of the Radiation Patterns of Simple HF Field Antennas in Open (Level) Terrain, Mountains and Forests," Special Technical Report 45 Stanford Research Institute, Menlo Park CA, Dec. 1971. 


\section{APPENDIX}

\section{Transformation of an Antenna from Horizontal Ground to the Terrain Suriace}

While irregular terrain may have important effects on antenna radiation, the current distribution of the antenna on a locally smooth surface may be close to that for the same antenna on flat ground. With this assumption, the antenna currents can be determined with a code such as NEC that will model antennas on a flat ground plane. Since the ground plane in NEC is the $x-y$ plane, the antenna currents must be transformed to the terrain surface and reoriented with respect to the local terrain normal. One way to accomplish this transformation might be with a CAD program under user control. However, a predetermined transformation could give a more accurate orientation, and would be essential without a CAD system.

For the transfo:mation given here it is assumed that the antenna is modeled in the VEC coordinate system on a ground surface in the $x-y$ plane and is located with respect to the origin of that coordinate system. The antenna is transformed to a specified point $\left(x_{t}, y_{t}\right)$ in the terrain coordinate system with the $z$ direction in the NEC coordinates being aligned with the local normal on the terrain. In addition, a line specified by the angle $\phi_{\ell}$ in the $x-y$ plane of the NEC coordinates will lie on a level contour of the terrain surface, pointing to the right when an observer faces down hill.

If the terrain surface is defined as $z=h(x, y)$ the unit normal vector is

$$
\begin{aligned}
\vec{n}(x, y) & =-\frac{\partial h(x, y)}{\partial x} \hat{x}-\frac{\partial h(x, y)}{\partial y} \hat{y}+\hat{z} \\
\hat{n}(x, y) & =\vec{n} /|\vec{n}|=n_{x}(x, y) \hat{x}+n_{y}(x, y) \hat{y}+n_{z}(x, y) \hat{z} \\
& =\sin \theta_{n} \cos \phi_{n} \hat{x}+\sin \theta_{n} \sin \phi_{n} \hat{y}+\cos \theta_{n} \hat{z} .
\end{aligned}
$$

Then to transform the NEC origin to the point $\left(x_{t}, y_{t}, z_{t}\right)$ on the terrain surface, where $z_{t}=h\left(x_{t}, y_{t}\right)$, define

$$
\begin{aligned}
C_{\ell} & =\cos \phi_{\ell} \\
S_{\ell} & =\sin \phi_{\ell} \\
C_{\theta} & =\cos \theta_{n}=n_{z}\left(x_{t}, y_{t}\right) \\
S_{\theta} & =\sin \theta_{n}=\sqrt{1-n_{z}^{2}\left(x_{t}, y_{t}\right)} \\
C_{\phi} & =\cos \phi_{n}=n_{x}\left(x_{t}, y_{t}\right) / \sin \theta_{n} \\
S_{\phi} & =\sin \phi_{n}=n_{y}\left(x_{t}, y_{t}\right) / \sin \theta_{n} .
\end{aligned}
$$

The NEC coordinates are first rotated so that the line defined by $\phi_{\ell}$ lies along the negative $y$ axis. Thus the point $\left(x_{0}, y_{0}, z_{0}\right)$ is transformed as

$$
\left(\begin{array}{l}
x_{1} \\
y_{1} \\
z_{1}
\end{array}\right)=\left(\begin{array}{ccc}
-S_{\ell} & C_{\ell} & 0 \\
-C_{\ell} & -S_{\ell} & 0 \\
0 & 0 & 1
\end{array}\right)\left(\begin{array}{l}
x_{0} \\
y_{0} \\
z_{0}
\end{array}\right) .
$$


Next the $z$ axis is rotated toward $\mathrm{x}$ by $\theta_{n}$

$$
\left(\begin{array}{l}
x_{2} \\
y_{2} \\
z_{2}
\end{array}\right)=\left(\begin{array}{ccc}
C_{\theta} & 0 & S_{\theta} \\
0 & 1 & 0 \\
-S_{\theta} & 0 & C_{\theta}
\end{array}\right)\left(\begin{array}{l}
x_{1} \\
y_{1} \\
z_{1}
\end{array}\right)
$$

and then the new $\mathrm{x}$ axis is rotated toward $\mathrm{y}$ by $\phi_{n}$

$$
\left(\begin{array}{l}
x_{3} \\
y_{3} \\
z_{3}
\end{array}\right)=\left(\begin{array}{ccc}
C_{\phi} & -S_{\phi} & 0 \\
S_{\phi} & C_{\phi} & 0 \\
0 & 0 & \mathrm{i}
\end{array}\right)\left(\begin{array}{l}
x_{2} \\
y_{2} \\
z_{2}
\end{array}\right)
$$

Combining these rotations with a translation to the point $\left(x_{t}, y_{t}, z_{t}\right)$ on the terrain surface. the point $\left(x_{0}, y_{0}, z_{0}\right)$ in the NEC coordinates transforms to the point $\left(x_{a}, y_{a}, z_{a}\right)$ in the terrain coordinates where

$$
\left(\begin{array}{c}
x_{a} \\
y_{a} \\
z_{a}
\end{array}\right)=\left(\begin{array}{ccc}
-C_{\phi} C_{\theta} S_{\ell}+S_{\phi} C_{\ell} & C_{\phi} C_{\theta} C_{\ell}+S_{\phi} S_{\ell} & C_{\phi} S_{\theta} \\
-S_{\phi} C_{\theta} S_{\ell}-C_{\phi} C_{\ell} & S_{\phi} C_{\theta} C_{\ell}-C_{\phi} S_{\ell} & S_{\phi} S_{\theta} \\
S_{\theta} S_{\ell} & -S_{\theta} C_{\ell} & C_{\theta}
\end{array}\right)\left(\begin{array}{c}
x_{0} \\
y_{0} \\
z_{0}
\end{array}\right)+\left(\begin{array}{c}
x_{t} \\
y_{t} \\
z_{t}
\end{array}\right) .
$$



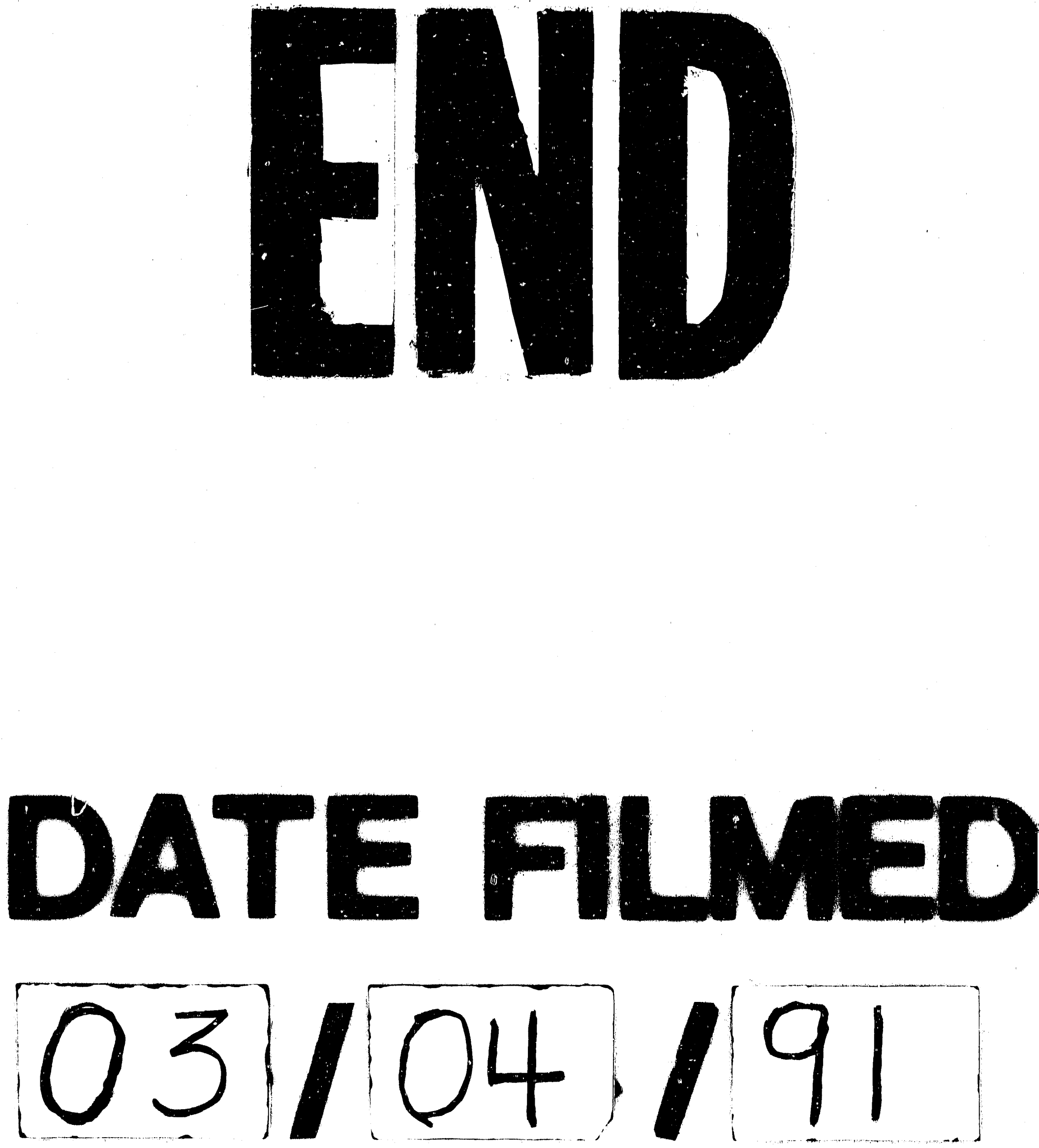
